\title{
Barotropic Regeneration of Upper-Level Synoptic Disturbances in Different Configurations of the Zonal Weather Regime
}

\author{
GWENDAL RiVIÈRE \\ Groupe d'Etude de l'Atmosphère Météorologique, CNRS and Météo-France, Toulouse, France
}

(Manuscript received 29 August 2007, in final form 18 December 2007)

\begin{abstract}
Barotropic dynamics of upper-tropospheric midlatitude disturbances evolving in different configurations of the zonal weather regime (i.e., in different zonal-like large-scale flows) were studied using observational analyses and barotropic model experiments.

The contraction stage of upper-level disturbances that follows their elongation stage leads to an increase of eddy kinetic energy that is called the barotropic regeneration process in this text. This barotropic mechanism is studied through notions of barotropic critical regions (BtCRs) and effective deformation that have been introduced in a previous paper. The effective deformation field is equal to the difference between the square of the large-scale deformation magnitude and the square of the large-scale vorticity. Regions where the effective deformation is positive correspond to regions where the large-scale flow tends to strongly stretch synoptic disturbances. A BtCR is an area separating two large-scale regions of positive effective deformation, one located upstream and on the south side of the jet and the other downstream and on the north side. Such a region presents a discontinuity in the orientation of the dilatation axes and is a potential area where the barotropic regeneration process may occur.

Winter days presenting a zonal weather regime in the 40-yr ECMWF Re-Analysis dataset are decomposed, via a partitioning algorithm, into different configurations of the effective deformation field at 300 $\mathrm{hPa}$. A six-cluster partition is obtained. Composite maps of the barotropic generation rate for each cluster exhibit a succession of negative and positive values on both sides of the BtCRs. It confirms statistically that the barotropic regeneration mechanism occurs preferentially about BtCRs.

Numerical experiments using a forced barotropic model on the sphere are performed. Each experiment consists of adding a synoptic-scale perturbation to one of the zonal-like jet configurations found in observations, which is kept fixed with time. The combined effects of the effective deformation and nonlinearities are shown to be crucial to reproduce the barotropic regeneration process about BtCRs.
\end{abstract}

\section{Introduction}

Synoptic disturbances in the upper troposphere take the form of coherent wave packets with particularly intense mobile troughs surrounding the globe in the midlatitudes and play a crucial role in the dynamics of various weather systems. First, they are involved in the triggering of extratropical surface cyclogenesis via baroclinic interaction (Petterssen and Smebye 1971) and second, they act on the large-scale atmospheric circulation through eddy momentum fluxes (Hoskins et al. 1983). In other words, they and surface cyclones to-

Corresponding author address: Gwendal Rivière, MétéoFrance, CNRM/GMAP/RECYF, 42 av. G. Coriolis, 31057 Toulouse CEDEX 1, France.

E-mail: gwendal.riviere@meteo.fr gether constitute the two main components of the socalled storm tracks, and their life cycles are thus particularly important to document and analyze. Among the processes involved in the dynamics of uppertropospheric disturbances are baroclinic interaction, downstream development, and barotropic modulation (e.g., Nielsen-Gammon and Lefevre 1996; Chang et al. 2002). The aim of the present study is to focus on the latter process, that is, on the barotropic effects resulting from the horizontal variations of the background largescale flow.

The climatology study of Sanders (1988) was among the first to notice that upper-level mobile troughs are usually generated east of major mountain chains (e.g., east of the Rocky Mountains in North America and east of the Himalayas in Asia). Their births are particularly favored in the presence of a northwesterly flow 
over these regions (Schultz and Sanders 2002). By contrast, the deaths of upper-level mobile troughs occur preferentially over the oceans. Lackmann et al. (1997, 1999) and, more recently, Schultz and Sanders (2002) have shown that the genesis of upper-tropospheric troughs east of the mountains can be explained by barotropic processes. An initially elongated trough evolving in a northwesterly flow, say over North America, is rapidly compacted east of the Rockies, leading to a deepening of the trough and an increase of eddy kinetic energy. This mechanism is confirmed by the climatological maps of barotropic generation rate made independently by Lee (2000) and Black and Dole (2000). The barotropic generation rate characterizes the amount of kinetic energy extracted by the eddies from the background flow; it is positive east of the mountains and strongly negative over the Pacific and Atlantic, leading to an increase and a loss, respectively, of eddy kinetic energy in those regions. Lee (2000) explains this result by the fact that meridionally elongated waves in the diffluent region of the Pacific jet, for example, are contracted when they arrive in the confluent region at the entrance of the Atlantic jet. Furthermore, another area of positive barotropic generation rate exists over the North African subtropical jet; eddies evolving along the extratropical Atlantic jet get a northeast-southwest tilt in its exit region, propagate equatorward, and are compacted on the cyclonic side of the subtropical jet. Following all these studies, the elongation-contraction process (called hereafter the barotropic regeneration process) takes place preferentially east of the mountains in a confluent region or north of the subtropical jets. It is worth noting that this process is a transient nonmodal phenomenon that may occur without the presence of barotropic instability, as shown theoretically in simple linear barotropic models by Farrell (1989) and Lee (1995).

The transition from elongation to contraction stages requires a rapid change of the environment in which disturbances are embedded and, in particular, of the orientation of the dilatation axes. This is the case from a diffluent to a confluent region or from the anticyclonic side of the eddy-driven jet to the cyclonic side of the subtropical jet. Despite the lack of climatological evidences of such process over the ocean, case studies of Rivière and Joly (2006a, hereafter RJ06a) show that it may also occur sometimes over the Atlantic when an upper disturbance crosses the extratropical jet from the south to the north. This crossing takes place in specific regions of the large-scale extratropical flow called barotropic critical regions (BtCRs). Following theoretical results of Rivière et al. (2003, hereafter RHK03), RJ06a define a new quantity called effective deformation that is based on the stretching, shearing, and vorticity components of the large-scale deformation tensor. This quantity is shown to be more relevant than the classical deformation magnitude to analyzing deformation effects. A perturbation is strongly stretched in a region of strongly positive effective deformation but not necessarily in a region of large deformation magnitude. BtCRs are defined from horizontal maps of the effective deformation field. They separate two large-scale regions of positive effective deformation and are characterized by a rapid change of the dilatation axes' orientation. Note finally that BtCRs are shown in RJ06a to play an important role in the regeneration of both upper-level synoptic disturbances and surface cyclones.

The notions of weather regimes have been introduced by Vautard (1990) to represent quasi-stationary circulations of the large-scale atmospheric flow. Four weather regimes are identified in the Atlantic: the Atlantic ridge, the zonal regime, the Greenland anticyclone, and the blocking. The zonal regime corresponds to a zonal-like jet along the latitude $45^{\circ} \mathrm{N}$. Two distinct periods corresponding both to a zonal weather regime are studied in RJ06a. Despite the apparent similarities between the two zonal-like jets, they present very different configurations of the effective deformation fields. For one jet (mid-February 1997), a BtCR and its associated barotropic regeneration process is present at the jet exit, whereas for the other (end of December 1999) it is at the jet entrance only. Two apparently similar zonal jets lead therefore to very different behavior of the synoptic eddies. The aim of the present study is to validate these results statistically and to analyze the different configurations of the zonal weather regime systematically, in terms of their effective deformation fields and their impact on synoptic uppertropospheric disturbances. To perform this statistical study, 40-yr European Centre for Medium-Range Weather Forecasts (ECMWF) Re-Analysis (ERA-40) data are used and analyzed for all the winter periods from 1957 to 2001 . The second purpose of the study is to reproduce the same mechanism shown in reanalysis data in idealized barotropic simulations and to identify key factors that explain the whole barotropic regeneration process about a BtCR.

The paper is divided as follows: Barotropic dynamics related to the notions of effective deformation field and barotropic critical regions are briefly recalled in section 2. Section 3 is dedicated to the observations of the different configurations of the zonal weather regime. The dynamical cluster algorithm of Michelangeli et al. (1995, hereafter MVL95) is introduced to separate zonal weather regimes into different configurations of the effective deformation field at $300 \mathrm{hPa}$. In the same 
section, a statistical analysis of the barotropic regeneration process about a BtCR is described. In section 4, numerical experiments in a forced barotropic model on the sphere are performed. Each experiment consists of adding a synoptic-scale perturbation initially to one of the zonal-like jet configurations found in section 3 that is maintained fixed during the whole simulation. Section 4 completes the results of section 3 and shows the crucial importance of the combined effects of the effective deformation and the nonlinear terms to reproduce the barotropic regeneration process about a BtCR. Conclusions are presented in section 5 .

\section{Barotropic dynamics}

\section{a. Barotropic generation rate and effective deformation}

The following theoretical aspects are described in detail in RHK03 within the quasigeostrophic framework. They have been then applied to the real atmosphere in RJ06a and are briefly recalled in this section.

The barotropic energy exchanges between the highfrequency component (denoted with primes) and the low-frequency component (denoted with subscript $m$ ) of the atmospheric flow are usually diagnosed by the so-called barotropic generation rate, which can be expressed following Mak and Cai (1989) as the scalar product $\mathbf{E} \cdot \mathbf{D}_{\mathbf{m}}$, where

$$
\begin{aligned}
\mathbf{E} \equiv & {\left[\frac{1}{2}\left(v^{\prime 2}-u^{\prime 2}\right),-u^{\prime} v^{\prime}\right] \text { and } } \\
\mathbf{D}_{\mathbf{m}} \equiv & \left(\partial u_{m} / \partial x-\partial v_{m} / \partial y-v_{m} \tan \varphi / a, \partial v_{m} / \partial x\right. \\
& \left.+\partial u_{m} / \partial y+u_{m} \tan \varphi / a\right) .
\end{aligned}
$$

The variables $u$ and $v$ are the horizontal velocity components, $\varphi$ is latitude, $a$ is the earth's radius, $\partial / \partial x \equiv(a$ $\cos \varphi)^{-1} \partial / \partial \lambda$ and $\partial / \partial y \equiv a^{-1} \partial / \partial \varphi$ are the horizontal derivatives, and $\lambda$ is longitude. When $\mathbf{E} \cdot \mathbf{D}_{\mathbf{m}}$ is positive (negative), the perturbation extracts energy from (loses energy to) the reference flow. The barotropic generation rate is maximum (minimum) when the perturbation velocity vector aligns with the contraction (dilatation) axis. This leads to $\mathbf{E} \cdot \mathbf{D}_{\mathbf{m}}=+K_{e}^{\prime} \sigma_{m}\left(-K_{e}^{\prime} \sigma_{m}\right)$, where $\sigma_{m} \equiv\left|\mathbf{D}_{\mathbf{m}}\right|$ is the deformation magnitude and $K_{e}^{\prime} \equiv 1 / 2\left(u^{\prime 2}+v^{\prime 2}\right)$ is the perturbation kinetic energy.

However, the dilatation-contraction axes are not usually the equilibrium axes along which the perturbation orientation tends to align. In fact, two equilibrium axes exist whose exact formulations are provided in RHK03; one induces perturbation kinetic energy growth and is an unstable equilibrium, whereas the other induces perturbation decay and is stable. These orientations lead to the following approximate expression of the barotropic generation rate in the case of low-frequency flows with weak curvatures: $\mathbf{E} \cdot \mathbf{D}_{\mathbf{m}}=$ $\pm K_{e}^{\prime} \sqrt{\Delta_{m}}$. Here, $\Delta_{m}$ is called effective deformation and is defined as

$$
\Delta_{m} \equiv \sigma_{m}^{2}-\xi_{m}^{2},
$$

where $\xi_{m} \equiv \partial v_{m} / \partial x-\partial u_{m} / \partial y-u_{m} \tan \varphi / a$ is the rotation rate relative to the basis set $(i, j)(i$ and $j$ being the unit vectors directed eastward and northward). The sign of $\Delta_{m}$ is crucial because it determines regions where equilibrium axes exist and where they do not. Positive values of $\Delta_{m}$ correspond to regions where synoptic eddies can be strongly stretched by the deformation action and where barotropic processes can be very important. Note in particular that in the extreme case where $\Delta_{m} \gg$ 0 , the equilibrium axes are close to the dilatationcontraction axes. By contrast, in regions of negative $\Delta_{m}$ the rotation of the large-scale flow is so strong that the perturbation cannot be elongated along specific orientations. In the latter environment, the perturbation main axis keeps rotating and on average, barotropic processes are quite weak.

\section{b. Barotropic critical regions}

Because the stable equilibrium axis leads to destruction of kinetic energy, the high-frequency kinetic energy necessarily decreases after a while if the environment of the high-frequency eddy does not change. Barotropic development has, therefore, very good chances to occur when there is a rapid change of the environment, which can happen around barotropic critical regions (BtCR). A BtCR is defined as a specific area separating two regions of strongly positive effective deformation where the dilatation axes (or the stable equilibrium axes) are almost perpendicular upstream and downstream of it. The mechanism about a BtCR is as follows: Upstream of this area, the eddy is strongly stretched along the dilatation axes and loses energy. As it crosses the BtCR, its orientation becomes perpendicular to the new dilatation axes and it temporarily gains energy slightly downstream of the BtCR. After evolving in this new environment for a while, it is stretched again and loses energy. The aim of next section is to prove that this mechanism is statistically relevant.

\section{Observations of different configurations of zonal weather regimes}

\section{a. Data}

The data used in this section are the geopotential and the horizontal wind components extracted from the 
ERA-40 data for 1957-2001. The fields are interpolated on a longitude-latitude grid with $1.5^{\circ}$ horizontal resolution; only daily instantaneous maps at 1200 UTC during the winter period (16 October-15 April) are considered. In what follows, the effective deformation is computed from the low-frequency horizontal wind components by using centered finite differences and Eqs. (2) and (3). Low- and high-frequency components of the flow correspond hereafter to periods greater and less than 10 days, respectively, and have been obtained from a Lanczos temporal filter.

\section{b. Methodology}

The principles of the dynamical cluster algorithm used in this section are briefly recalled here, but more details are provided in MVL95. In what follows, the algorithm is applied to two different atmospheric fields: the geopotential and the effective deformation fields.

\section{1) Dynamical cluster algorithm}

Before using the partitioning algorithm, an EOF analysis is performed on a given field $X$ in a given domain to reduce the number of degrees of freedom. The aim is to choose an appropriate number of EOFs that explain a sufficient part of the total variance of $X$. For example, if $X$ is the geopotential field, 20 EOFs are enough to explain about $95 \%$ of the variance. The partitioning algorithm is then applied to the components of the field $X$ in this reduced EOF subspace whose dimension is much smaller than the gridpoint space.

Let us now define the partitioning algorithm itself. Given a number of clusters $k$, the principle of the algorithm is to partition the sample of fields considered into $k$ clusters such that the sum of the variances inside each cluster is minimized. The iterative algorithm starts with $k$ random fields chosen among the data and looks for a minimization of the sum of the variances. The minimum found is generally not the global one but is close to it. Because the result of each partition depends on the initial random fields, this step is repeated a few times (50 times, as in MVL95) and the best partition is chosen among them.

The next step is to define a classifiability index to find the right number of clusters $k$ as described in MVL95 and Plaut and Simonnet (2001). The classifiability index $c(k)$ is a measure estimating the resemblance of the different partitions obtained with the different random initializations. When this quantity is close to 1 , it means that the different partitions are close to each other. The right number of clusters is not, however, that leading to the largest classifiability index because $c(k)$ usually decreases with $k$ for any sample. The best way is to compare for each $k$ the classifiability index of the sample we wish to partition with the classifiability indexes obtained from randomly built samples. We then choose the index $c(k)$ that is significantly greater than the classifiability indexes of the random samples.

\section{2) Classification into FOUR WEATHER REgimes}

The previously described algorithm has been applied to the low-frequency geopotential field at $500 \mathrm{hPa}$ for all the winter days of the period 1958-2001 (i.e., to 7837 days) in the Atlantic domain $28.5^{\circ}-79.5^{\circ} \mathrm{N}, 79.5^{\circ} \mathrm{W}-$ $28.5^{\circ} \mathrm{E}$. By applying the test based on the classifiability index, $k=4$ and $k=8$ were found to be the two best suitable numbers of clusters $k$. Note that $k=4$ was also the number found by MVL95 and Plaut and Simonnet (2001) using the same method but applied to other datasets; $k=4$ is the number chosen in the present study. It leads to the so-called four weather regimes described initially by Vautard (1990): the Atlantic ridge, the zonal regime, the Greenland anticyclone, and the blocking, which represent respectively 1856, 2364, 1709, and 1908 days (see Figs. 1a-d).

\section{3) Classification of the efFective DEFORMATION FIELDS FOR THE ZONAL REGIME}

Our aim is to focus on the zonal regime and to find different configurations in terms of the effective deformation field. We apply to the 2364 days of the zonal regime the same cluster algorithm, but on the effective deformation field at $300 \mathrm{hPa}$. This level is chosen because it corresponds to the upper troposphere where the low-frequency jet and its horizontal gradients are stronger; $500 \mathrm{hPa}$ has been also tested and does not change the result of the partition. The number of EOFs needed to represent a sufficient part of the variance of the effective deformation field is much larger than in the case of the geopotential because the former is a much smaller-scale field than the latter. In our study, 100 EOFs are kept and represent $85 \%$ of the total variance. The classifiability index obtained after applying the partitioning algorithm is shown in Fig. 2. The two best choices for the number of clusters are $k=2$ and $k=6$ because for these specific values of $k$ the classifiability index of our sample (denoted with a circle) is significantly larger than that computed from randomly built samples (shaded areas). We keep $k=6$ to have stronger correlations inside each cluster and also because $k=2$ leads systematically to a very high classifiability index, which is usually artificial (see MVL95). 

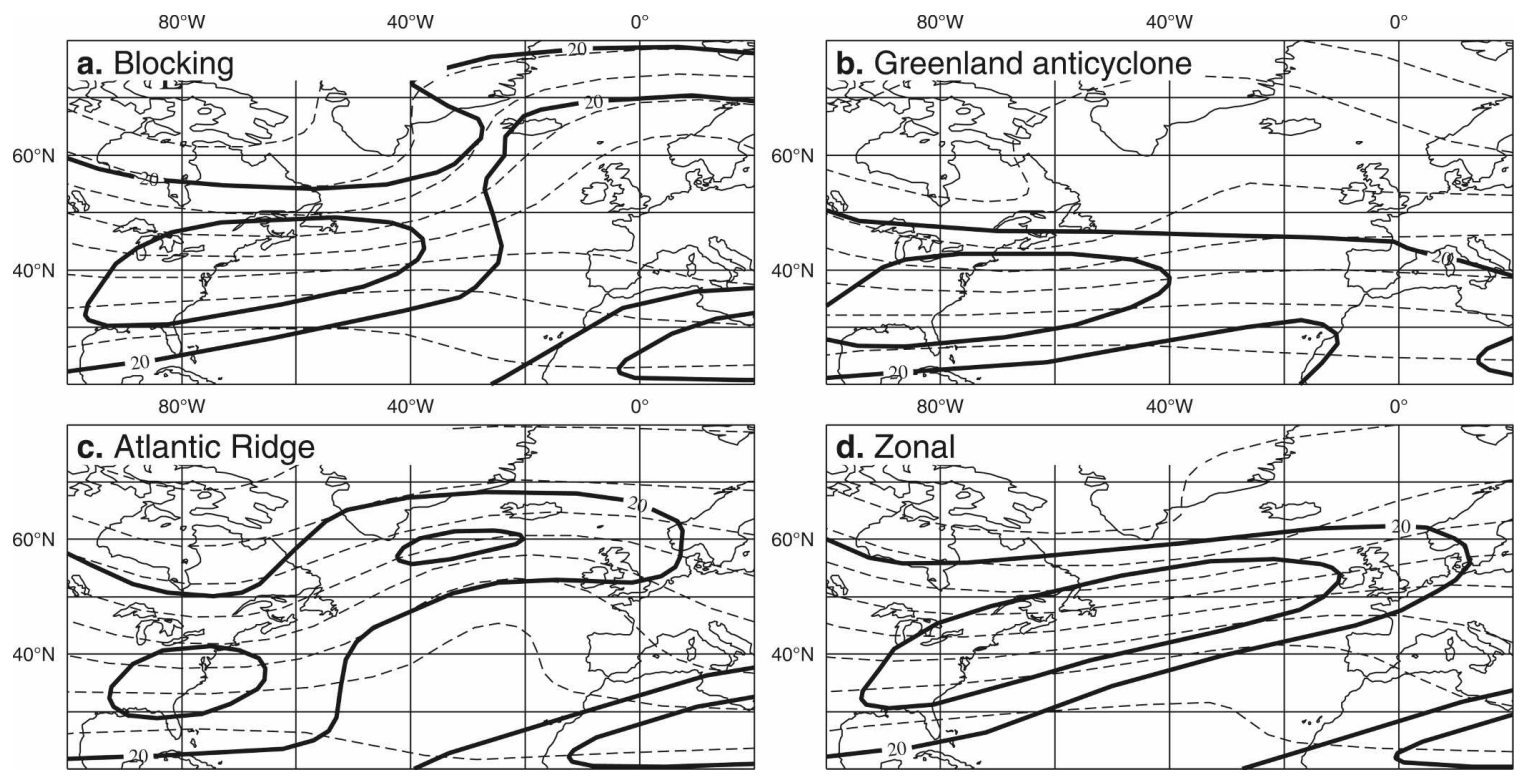

FIG. 1. Composites of the low-frequency geopotential field at $500 \mathrm{hPa}$ (dashed contours; intervals of $10^{3} \mathrm{~m}^{2} \mathrm{~s}^{-2}$ ) and of the low-frequency wind speeds at $300 \mathrm{hPa}$ (heavy black solid lines; intervals of $10 \mathrm{~m} \mathrm{~s}^{-1}$ for values larger than $20 \mathrm{~m} \mathrm{~s}^{-1}$ ) for the four-cluster geopotential partition: (a) blocking, (b) Greenland anticyclone, (c) Atlantic ridge, and (d) zonal regime.

\section{c. Composite patterns}

The present section is dedicated to the presentation of the six-cluster partition of the zonal regime. Centroids of the different clusters (i.e., composites of the effective deformation fields) are shown in Fig. 3. A succession of well-separated large-scale regions of positive effective deformation values is clearly visible along the low-frequency extratropical jet for all clusters. Approximately two or three such large-scale regions usually appear from the jet entrance to the jet exit depending on the cluster. Such a characteristic of the effective deformation field has been already noticed in case studies of RJ06a and Rivière and Joly (2006b). The bottleneck areas that separate two regions of positive $\Delta_{m}$ along the jet and that exhibit a discontinuity in the dilatation axes' orientation are BtCRs; they are automatically detected by the algorithm described in the appendix and numbered in Fig. 3. All the other bottleneck areas formed by the effective deformation outside the extratropical jet are not considered in the present study and are not detected by the algorithm. Clusters 1 , 2, 3, 4, and 5 have two numbered BtCRs, whereas cluster 6 has only one. The two zonal regimes studied in RJ06a (mid-February 1997 and late December 1999) are respectively included in clusters 1 and 6 . As noticed in the previously mentioned paper, there are some jets that exhibit a BtCR at the jet exit and others that do not. In the present six-cluster partition, clusters 1, 2, 3, and 4 present a BtCR at the jet exit east of $40^{\circ} \mathrm{W}$; clusters 5 and 6 do not.
The effective deformation field is compared to the classical deformation magnitude for the particular case of cluster 6 (see shaded contours of $\sigma_{m}^{2}$ in Fig. 4 and $\Delta_{m}$ in Fig. 3f). Similarities exist-both fields reach their maximum values north of the jet between $40^{\circ}$ and $0^{\circ} \mathrm{W}$-but discrepancies are also present: strong values of $\sigma_{m}^{2}$ are visible in regions of negative effective deformation south of the jet between $50^{\circ}$ and $30^{\circ} \mathrm{W}$ (it is a region of strong vorticity because of the anticyclonic curvature of the jet at these longitudes). Furthermore, the BtCR 6.A in Fig. $3 \mathrm{f}$ is not a remarkable point of the field $\sigma_{m}^{2}$ and cannot be detected by this classical diagnostic.

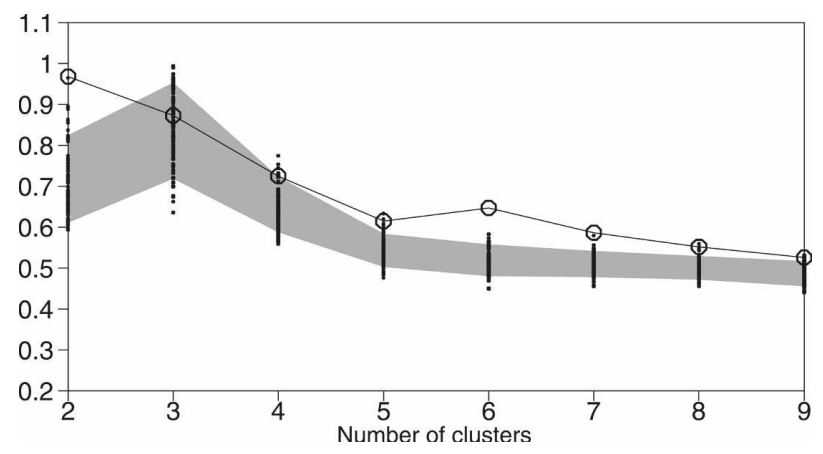

FIG. 2. The classifiability index for the dates of zonal regimes as a function of the number of clusters (solid line with circles). The shaded area represents the $10 \%-90 \%$ bounds of the classifiability index distribution calculated from randomly selected winter dates; 100 random samples of the same length are performed to build the $10 \%-90 \%$ bounds. More details are given in the text. 

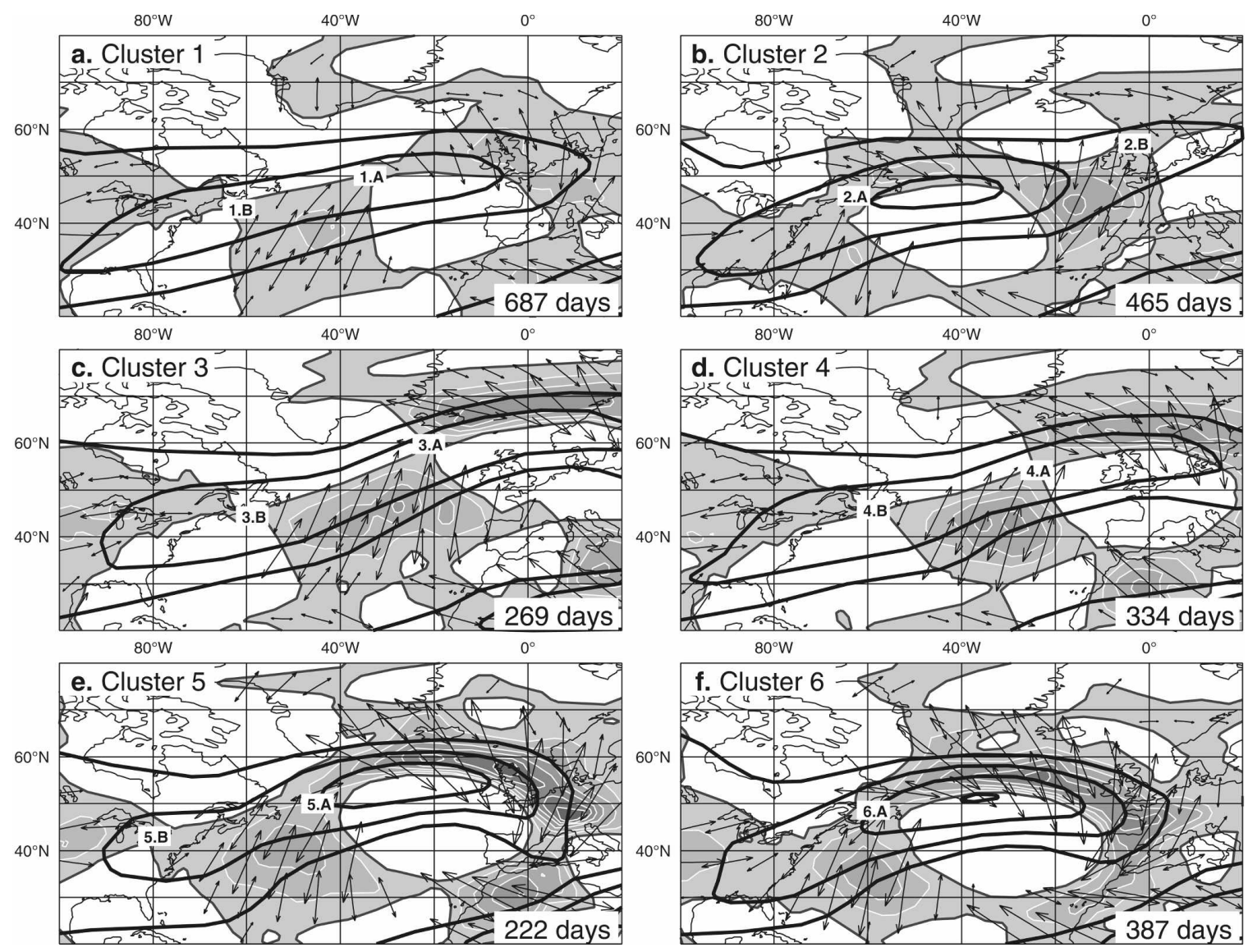

FIG. 3. Composites of the effective deformation fields $\Delta_{m}$ at $300 \mathrm{hPa}$ (gray shaded contours; intervals of $2.510^{-10} \mathrm{~s}^{-2}$ for positive values only) and of the wind speeds (heavy black solid lines; intervals of $10 \mathrm{~m} \mathrm{~s}^{-1}$ for values larger than $20 \mathrm{~m} \mathrm{~s}^{-1}$ ) for the six-cluster partition. Arrows correspond to composites of the dilatation axes in regions of positive $\Delta_{m}$. The number of days inside each cluster is given in the bottom right-hand corner of each panel. Barotropic critical regions are also indicated and numbered for each cluster.

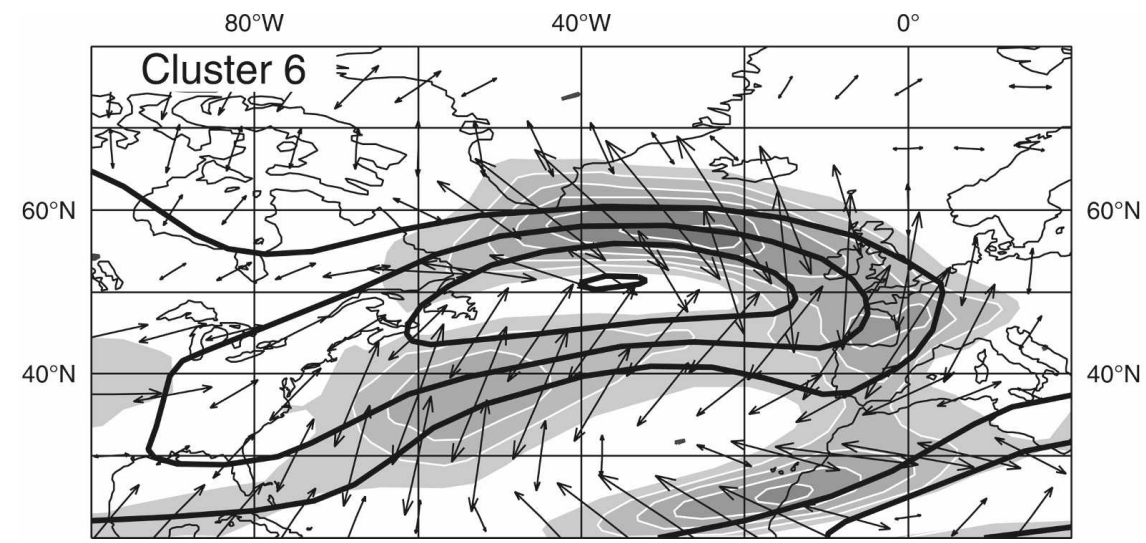

FIG. 4. Composite of the square of the deformation magnitude $\sigma_{m}^{2}$ at $300 \mathrm{hPa}$ (gray shaded contours; intervals of $2.510^{-10} \mathrm{~s}^{-2}$ for values greater than $2.510^{-10} \mathrm{~s}^{-2}$ ) and of the dilatation axes (black arrows) for the cluster- 6 case. Composites of the wind speeds are also represented as in Fig. 3. 

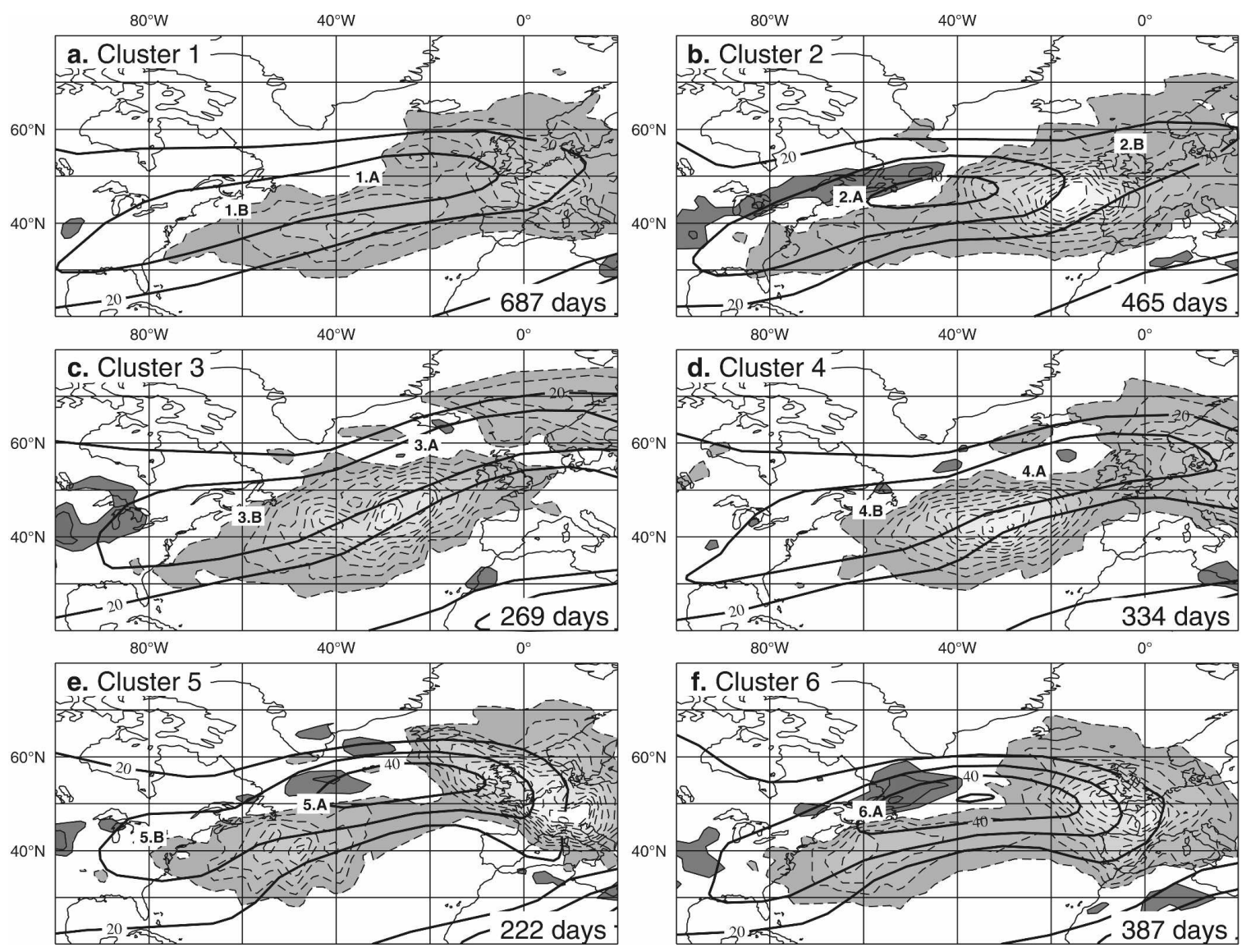

FIG. 5. Composites of the barotropic generation rate $\mathbf{E} \cdot \mathbf{D}_{\mathbf{m}}$ at $300 \mathrm{hPa}$ for the six-cluster partition (intervals of $510^{-4} \mathrm{~m}^{2} \mathrm{~s}^{-3} ;$ light shadings with dashed contours for negative values and dark shadings with solid contours for positive values). Composites of the wind speeds are also represented as in Fig. 3.

Figure 5 represents composites of the barotropic generation rate $\mathbf{E} \cdot \mathbf{D}_{\mathbf{m}}$ for the different clusters. Note first the presence of regions with systematically slight positive maxima of $\mathbf{E} \cdot \mathbf{D}_{\mathbf{m}}$ in each cluster. It concerns North America about the grid point $40^{\circ} \mathrm{N}, 90^{\circ} \mathrm{W}$, as well as north of the subtropical African jet (see the bottom right-hand part of each panel of Fig. 5). These two specific regions with systematic positive maxima of $\mathbf{E} \cdot \mathbf{D}_{\mathbf{m}}$ have been identified already in Lee (2000) as preferential zones for synoptic-eddy barotropic growth. Our purpose is now to analyze the differences among the different clusters. According to the schematic process involved about a BtCR, $\mathbf{E} \cdot \mathbf{D}_{\mathbf{m}}$ should be negative upstream of the BtCR, positive slightly downstream, and negative again far downstream. This characteristic is well visible about the BtCRs 2.A, 3.A, 4.A, 5.A, and 6.A even if the positive values slightly downstream of 3.A and 4.A are not very strong. Let us emphasize that the tripole pattern characterized by the succession of negative-positive-negative values of $\mathbf{E} \cdot \mathbf{D}_{\mathbf{m}}$ for cluster 6 can be interpreted by the spatial structure of the effective deformation field and its remarkable point 6.A but not at all by the spatial structure of the deformation magnitude shown in Fig. 4. However, note that no tripole pattern appears around 1.A, 1.B, 2.B, 3.B, 4.B, and 5.B.

To check the robustness of the results, other composites of $\mathbf{E} \cdot \mathbf{D}_{\mathbf{m}}$ have been computed by only keeping members that are close to their respective centroids. Because the dynamical cluster algorithm places each day of zonal regime in one particular cluster and does not reject any, the averaged correlations between the members and their centroids are not particularly high. They are equal to $0.22,0.34,0.45,0.42,0.53$, and 0.51 for clusters 1 to 6 , respectively. Note that cluster 1 is the cluster containing the largest number of members ( 687 days) and has a particularly weak intracorrelation. To eliminate members that do not resemble their centroids, composites of $\mathbf{E} \cdot \mathbf{D}_{\mathbf{m}}$ are made by only keeping days for which $\Delta_{m}$ has a correlation with its corresponding centroid stronger than 0.4 . With this more restrictive condition, cluster 1 contains only 105 days and its 

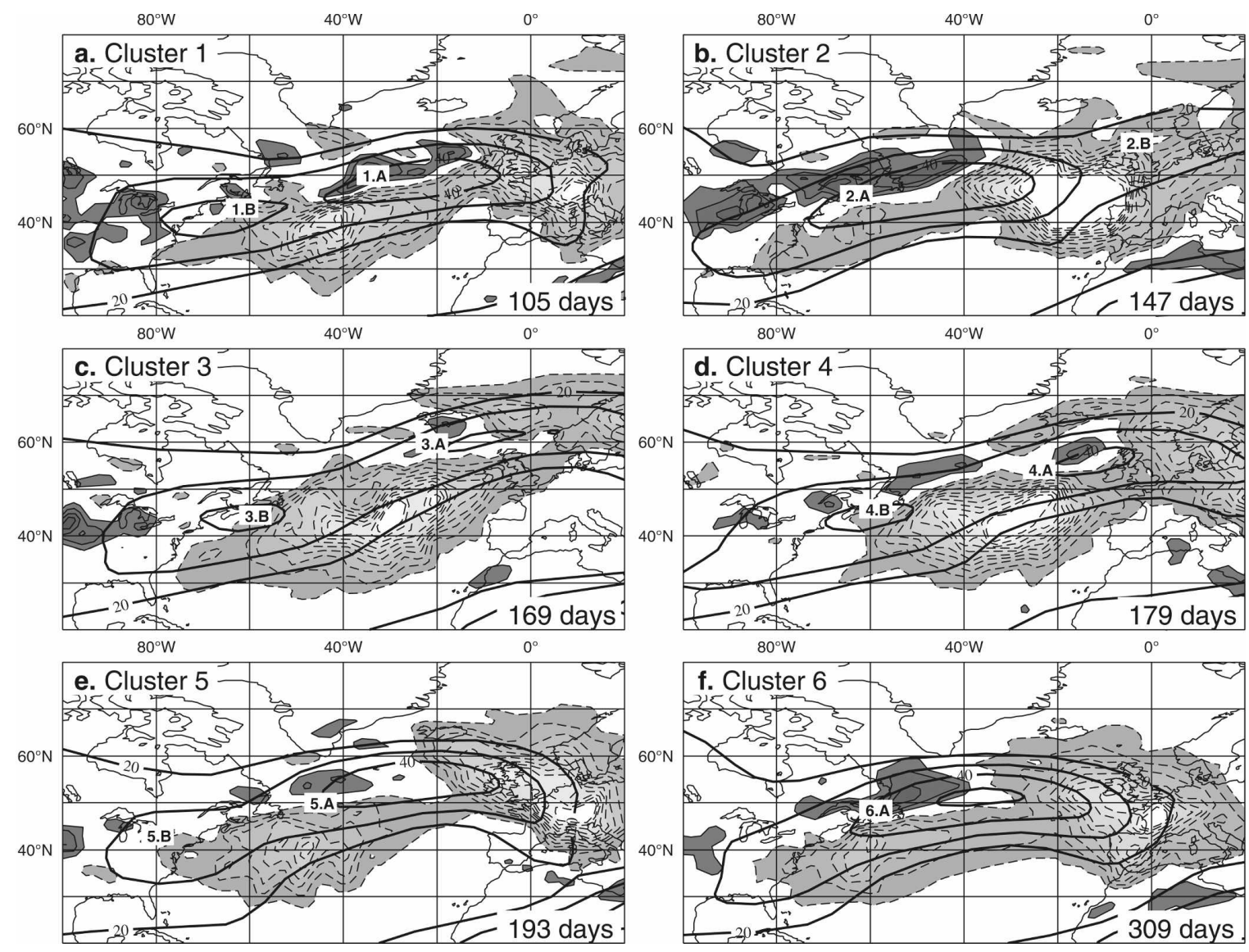

FIG. 6. As in Fig. 5, but the composites of $\mathbf{E} \cdot \mathbf{D}_{\mathbf{m}}$ are here made by considering only members having a correlation stronger than 0.4 with their respective centroids. The number of days used for each composite is given in the bottom right-hand corner of each panel. Composites of the wind speeds for the same samples are represented by heavy solid contours.

population has been divided by 6 , whereas populations of other clusters have been only divided by less than 2 . A succession of negative, positive, and negative values for $\mathbf{E} \cdot \mathbf{D}_{\mathbf{m}}$ is now visible about BtCR 1.A in Fig. 6a, which was not the case in Fig. 5a. This difference occurs precisely because most members of cluster 1 are too far from their centroid and do not present a BtCR about the point 1.A. Other composites of Fig. 6 are qualitatively not modified compared to Fig. 5; the tripole patterns around 2.A, 3.A, 4.A, 5.A, and 6.A are still there and are even more pronounced, but no such pattern is visible around 1.B, 2.B, 3.B, 4.B, and 5.B. We conclude from Figs. 5 and 6 that BtCRs denoted with letter $A$ exhibit the barotropic regeneration process whereas those denoted with letter $B$ do not. The commonality among the former regions that is not present in the latter ones (apart from 2.B) is that they all separate a region of positive $\Delta_{m}$ located upstream and south of the jet from another one located downstream and north of the jet. The crossing from the south to the north about the BtCR seems therefore to be a crucial point in re- producing the barotropic regeneration process. This aspect will be analyzed in the next section. The exception to this rule is point 2.B, which exhibits a crossing of $\Delta_{m}$ from the south to the north without presenting evidences of the regeneration process (values of $\mathbf{E} \cdot \mathbf{D}_{\mathbf{m}}$ are very weak downstream of 2.B). This may be due to the fact that 2.B is located far downstream in the jetexit region and there is not much kinetic energy crossing this region (see Fig. 7b).

Composites of high-frequency eddy kinetic energy (EKE) for all clusters are presented in Fig. 7. Maxima of EKE are located in regions of strong effective deformation, which is logical because eddies are strongly stretched in regions of large values of $\Delta_{m}$ and start losing their energy when they reach these regions. For example, for clusters 2,5 , and 6 , their global maxima are located in the jet-exit region far downstream of the points 2.A, 5.A, and 6.A, almost at the same place as global maxima of $\Delta_{m}$. For clusters 1, 3, and 4, no clear global maxima of EKE appear; large values of EKE are more spread out along the jet, but their local maxima 

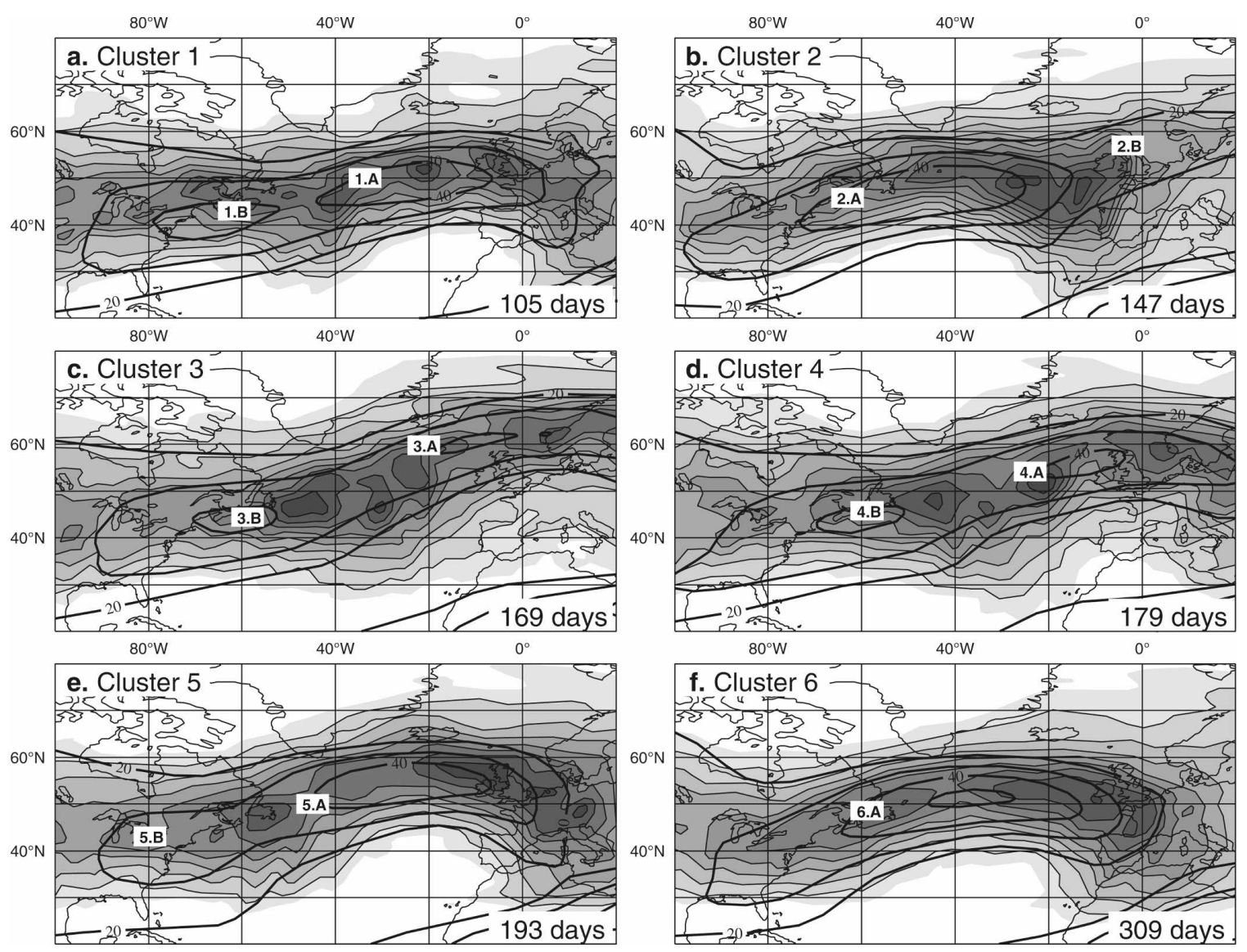

FIG. 7. Composites of the high-frequency eddy kinetic energy for the same days used in Fig. 6 (shaded contours; intervals of 30 $\mathrm{m}^{2} \mathrm{~s}^{-2}$ for values greater than $80 \mathrm{~m}^{2} \mathrm{~s}^{-2}$ ). Heavy solid contours as in Fig. 6.

are usually located in regions of strong $\Delta_{m}$. The elongation stages are also suggested by large values of EKE far south of the jet axis, in particular upstream of 1.A, 3.A, 4.A, and 5.A. Note, for example, the southward extension of EKE between longitudes $60^{\circ} \mathrm{W}$ and $40^{\circ} \mathrm{W}$ in Fig. $7 \mathrm{a}$, between $60^{\circ} \mathrm{W}$ and $20^{\circ} \mathrm{W}$ in Figs. $7 \mathrm{c}, \mathrm{d}$, and between $80^{\circ} \mathrm{W}$ and $40^{\circ} \mathrm{W}$ in Fig. 7e. High-frequency eddies are strongly stretched in these large-scale regions on the anticyclonic side of the jet where $\Delta_{m}$ is strongly positive, and their structures extend far south of the jet because of this stretching. It supports in particular the idea of a strong elongation stage occurring upstream of the BtCRs.

To conclude, statistical evidences of barotropic regeneration have been shown for some particular BtCRs (those denoted with letter $A$ ) but not for all of them. The aim of next section is to reproduce in a barotropic model the underlying barotropic regeneration mechanism to better understand what essential ingredient about a BtCR makes the occurrence of this mechanism possible.

\section{Simulations in a forced barotropic model}

\section{a. Model}

In the different numerical experiments, synopticscale perturbations are initially added to a basic state with a nontrivial effective deformation field. The basicstate streamfunction is the low-frequency streamfunction composite of one of the six clusters described in the previous section. Because the basic state is generally not stationary in our simplified model and its shape changes rapidly within few days, additional terms are added in the vorticity equation to make the basic-state stationary. The total streamfunction $\psi$ is the sum of a perturbation streamfunction $\psi^{\prime}$, and a basic state streamfunction $\bar{\psi}$ and the time evolution of $\psi^{\prime}$ is determined from the following nonlinear barotropic vorticity equation on the sphere:

$$
\begin{aligned}
\frac{\partial \nabla^{2} \psi^{\prime}}{\partial t}= & -J\left(\psi^{\prime}, \nabla^{2} \bar{\psi}+f\right)-J\left(\bar{\psi}, \nabla^{2} \psi^{\prime}\right)-J\left(\psi^{\prime}, \nabla^{2} \psi^{\prime}\right) \\
& -v \nabla^{10} \psi^{\prime},
\end{aligned}
$$




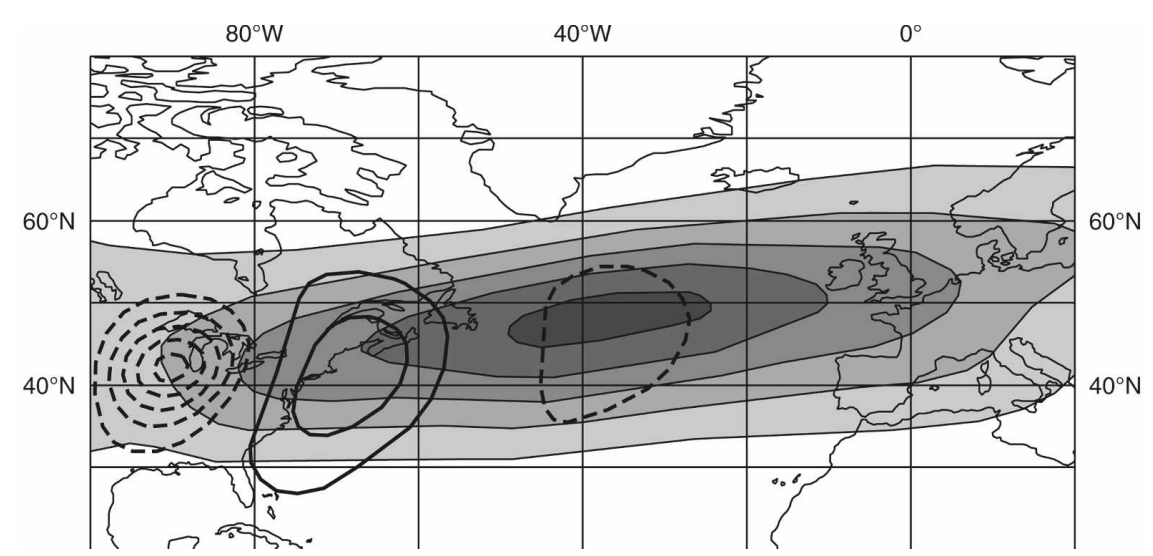

FIG. 8. Variance of the high-frequency streamfunction field at $300 \mathrm{hPa}$ (shaded contours; intervals of $2.510^{13} \mathrm{~m}^{4} \mathrm{~s}^{-2}$ for values larger than $5 \times 10^{13} \mathrm{~m}^{4} \mathrm{~s}^{-2}$ ) and regression of the same variable against the high-frequency vorticity at the base point $42^{\circ} \mathrm{N}, 90^{\circ} \mathrm{W}$ and at the same level (heavy solid and dashed contours indicate respectively positive and negative values; intervals of $\left.1 \times 10^{6} \mathrm{~m}^{2} \mathrm{~s}^{-1}\right)$. Each computation is done with the total number of days of zonal regimes (2364 days).

where $f$ denotes the Coriolis parameter. The model has a T81 resolution and $v=2 \times 10^{35} \mathrm{~m}^{8} \mathrm{~s}^{-1}$.

The barotropic generation rate is now denoted as $\mathbf{E} \cdot \overline{\mathbf{D}}$ to mark the difference from $\mathbf{E} \cdot \mathbf{D}_{\mathbf{m}}$ of the previous section, in which the decomposition eddy/mean flow was different and based on a time filtering method.

\section{b. Experiments}

Our experiments consist of initializing a perturbation upstream of the Atlantic domain and letting it evolve along the different configurations of jets. Two kinds of perturbations have been tested, one defined from reanalysis data and the other from analytical formula. The simulations are stopped after 3 days because most of the perturbation energy has crossed the Atlantic domain after this time.

\section{1) Perturbations from Reanalysis data}

The high-frequency streamfunction variance for the days exhibiting a zonal regime is shown in Fig. 8. At longitude $90^{\circ} \mathrm{W}$, the peak of the variance is around latitude $42^{\circ} \mathrm{N}$. The regression of the high-frequency streamfunction against the high-frequency vorticity at the base point $42^{\circ} \mathrm{N}, 90^{\circ} \mathrm{W}$ defines the perturbation for all simulations of this section (see the heavy solid and dashed contours in Fig. 8).

The results of six simulations having the same initial perturbation and differing in their basic states are shown in Fig. 9. Each basic-state streamfunction is defined as the composite of the low-frequency streamfunction of one of the six clusters described previously. Time averages between 0 and 3 days of $\mathbf{E} \cdot \overline{\mathbf{D}}$ are dis- played. Triangles represent BtCRs where the barotropic regeneration process has been shown to occur preferentially in the observations (those denoted with letter A). A good spatial correspondence exists between the simulations and the observations: the spatial correlations between $\mathbf{E} \cdot \overline{\mathbf{D}}$ of Fig. 9 and $\mathbf{E} \cdot \mathbf{D}_{\mathbf{m}}$ of Fig. 6 computed in the Atlantic domain are equal to 0.56, 0.65, $0.68,0.61,0.46$, and 0.46 for clusters 1 to 6 , respectively. The negative and positive values upstream and downstream of the triangles are clearly visible in all panels of Fig. 9 with more or less intensity. It confirms the occurrence of the barotropic regeneration mechanism about these specific regions in the simulations. Negative values far downstream of the BtCRs are also visible in all panels (except for Fig. 9c), indicating that the barotropic regeneration process is a transient phenomenon as expected from the theory. The maximum of perturbation kinetic energy is located more or less along the jet axis during each simulation (see heavy solid lines in Fig. 9), even if a slight displacement from the south to the north about each BtCR is visible. It suggests that the barotropic regeneration mechanism can occur without a frank crossing of the synoptic perturbations from the south to the north.

The time evolution of the perturbation embedded in the cluster-4 environment is presented in the left column of Fig. 10. The right column shows the same simulation but the perturbation amplitude has been initially divided by 10 . The aim is to distinguish between linear and nonlinear effects: simulations on the left and on the right are respectively called the nonlinear and linear runs. At day 0.5, there is no real difference between the two simulations (see Figs. 10a,b). Twelve hours later, at 

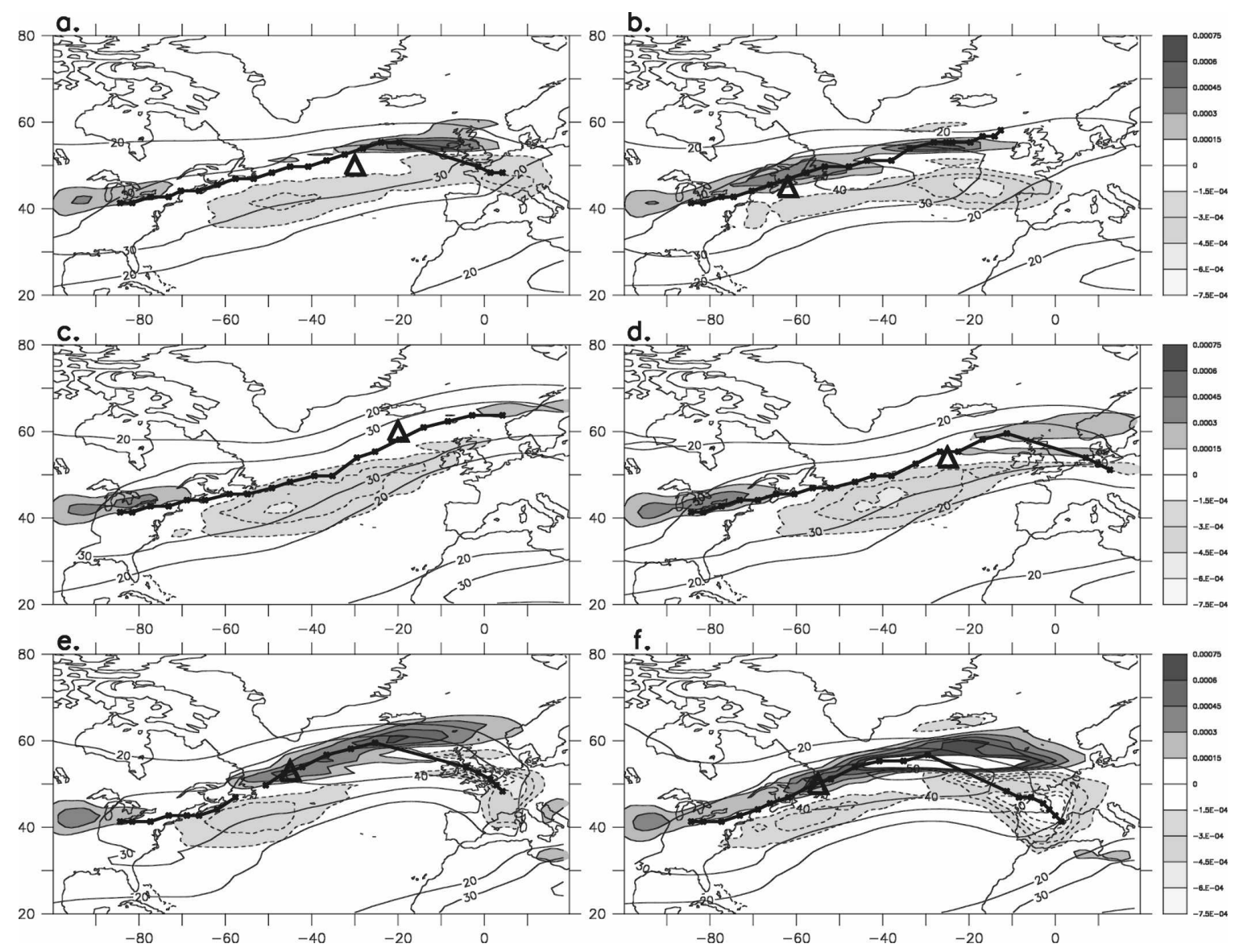

FIG. 9. Time averages of the barotropic generation rate $\mathbf{E} \cdot \overline{\mathbf{D}}$ (shaded contours; light shadings with dashed contours for negative values and dark shadings with solid contours for positive values) for six simulations differing from their basic states. Each flow is initially defined as the sum of a basic state and a perturbation; the basic state is the streamfunction composite of one of the six clusters and the perturbation is the regression shown in Fig. 8; (a)-(f) correspond to the basic states issued from clusters 1 to 6 , respectively. The perturbation streamfunction maximum is located at the base point $42^{\circ} \mathrm{N}, 90^{\circ} \mathrm{W}$ and equals $15 \times 10^{6} \mathrm{~m}^{2} \mathrm{~s}^{-1}$ for all simulations (i.e., slightly less than twice the standard deviation of the high-frequency streamfunction at the same point). For each simulation, the trajectory of the maximum of eddy kinetic energy is shown by heavy solid lines with crosses and the wind speed of the basic state by thin solid lines. Triangles indicate the BtCRs named with letter $A$ in Figs. 3-7.

day 1.0, the vorticity maximum in Fig. 10c is over Newfoundland whereas in Fig. 10d it is located south of Newfoundland. Furthermore, $\mathbf{E} \cdot \overline{\mathbf{D}}$ is essentially negative at that time for both simulations, with stronger negative values for the linear run (Fig. 10d). At day 1.5, the differences become more pronounced; the perturbation maximum is around the jet axis for the nonlinear run (Fig. 10e), whereas it is clearly located on the south side of the jet for the linear run (Fig. 10f). Both have still negative values of $\mathbf{E} \cdot \overline{\mathbf{D}}$ because of the stretching of the perturbations along the dilatation axes. Note that although the perturbation vorticity maximum is located around the jet axis in the nonlinear run, the perturbation (in particular the anticyclone) is elongated on the anticyclonic side of the jet in regions where $\Delta_{m}$ is strongly positive and gets a northeast-southwest tilt.
From days 1.5 to 2.0 , the vorticity maximum in the nonlinear run is rapidly pushed poleward (cf. Figs. $10 \mathrm{e}, \mathrm{g})$ during its crossing of the BtCR, and at day 2.0, $\mathbf{E} \cdot \overline{\mathbf{D}}$ is positive, with isolines separating the cyclone from the anticyclone perpendicular to the new dilatation axes' orientation. By contrast, at the same time, the perturbation in the linear run is farther southward; it did not cross the BtCR at all and still loses energy (Fig. 10h). The same results (not shown here) have been found for all the other clusters. To conclude, nonlinear effects are crucial to reproduce the barotropic regeneration mechanism about a BtCR.

The time average of $\mathbf{E} \cdot \overline{\mathbf{D}}$ for the nonlinear and linear runs are respectively shown in Figs. 11a,b. There are strong negative values southwest of the BtCR for both runs; however, no prominent signal appears far- 

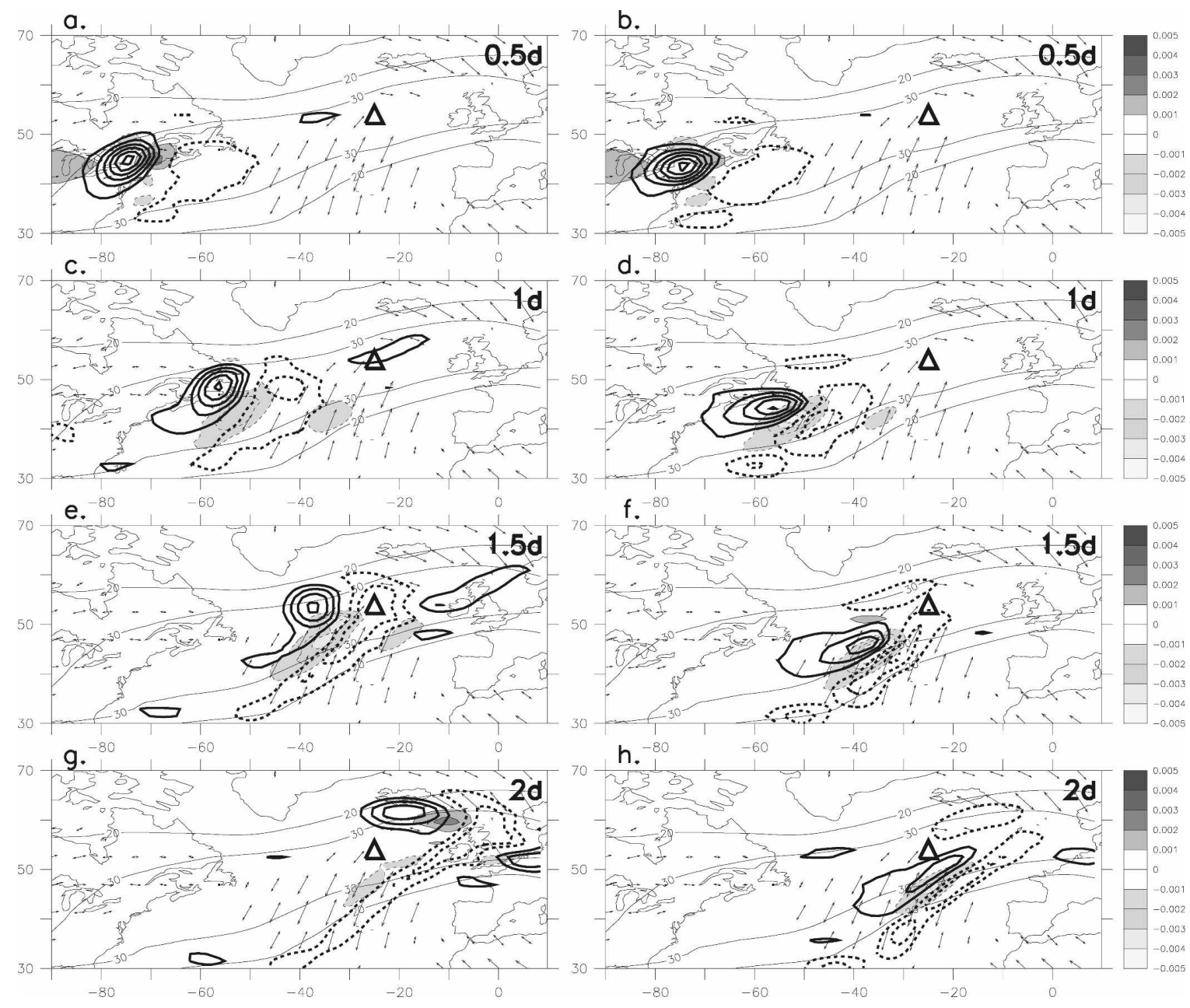

FIG. 10. Time evolution of the barotropic generation rate $\mathbf{E} \cdot \overline{\mathbf{D}}$ (shaded contours; light shadings with dashed contours for negative values and dark shadings with solid contours for positive values; intervals of $10^{-3} \mathrm{~m}^{2} \mathrm{~s}^{-3}$ ) and of the perturbation vorticity (intervals of $2 \times 10^{-5} \mathrm{~s}^{-1}$; heavy dashed contours for negative values and heavy solid contours for positive values) for an initial perturbation with maximum amplitude equal to (left) $15 \times 10^{6} \mathrm{~m}^{2} \mathrm{~s}^{-1}$ and (right) $15 \times 10^{5} \mathrm{~m}^{2} \mathrm{~s}^{-1}$. Arrows correspond to the dilatation axes in regions of positive $\Delta_{m}$ and thin solid lines to the basic-state wind speed; $\mathbf{E} \cdot \overline{\mathbf{D}}$ and the perturbation vorticity are respectively multiplied by 100 and 10 in the run on the right to make the comparison easier between the two runs. Both simulations are done with the cluster- 4 basic state; (a)-(b), (c)-(d), (e)-(f), and (g)-(h) correspond respectively to the flows 12, 24, 36 and $48 \mathrm{~h}$ after the initial time.

ther eastward for the linear run (Fig. 11b), whereas a positive maximum of $\mathbf{E} \cdot \overline{\mathbf{D}}$ is well visible northeast of the BtCR for the nonlinear run (Fig. 11a). It confirms the comments made in the previous paragraph. The time average of $\mathbf{E} \cdot \overline{\mathbf{D}}$ of another simulation is displayed in Fig. 11c. It is the same simulation as in Fig. 11a, but the sign of the initial perturbation has been reversed. By changing sign, the perturbation reaches its maximum amplitude in the center of an anticyclone and not of a cyclone as before. In Fig. 11c, the behavior is very different from Fig. 11a, with a tendency for the perturbation to go farther southward during the whole simulation, and there is no crossing of the BtCR. It suggests again the crucial role played by the nonlineari- ties. The barotropic regeneration mechanism can only occur in the presence of a cyclone with significant amplitude.

\section{2) AnAlytically PRESCRIBed PERTURbations}

In the previous section, perturbations are defined from linear regressions using reanalysis data. Because the perturbation spatial structure obtained from the regression map depends on the grid point considered and because we want to test the sensitivity to the location independently of the perturbation shape, it is more suitable to analytically define perturbations. It allows us to initialize the same perturbation at different locations. 


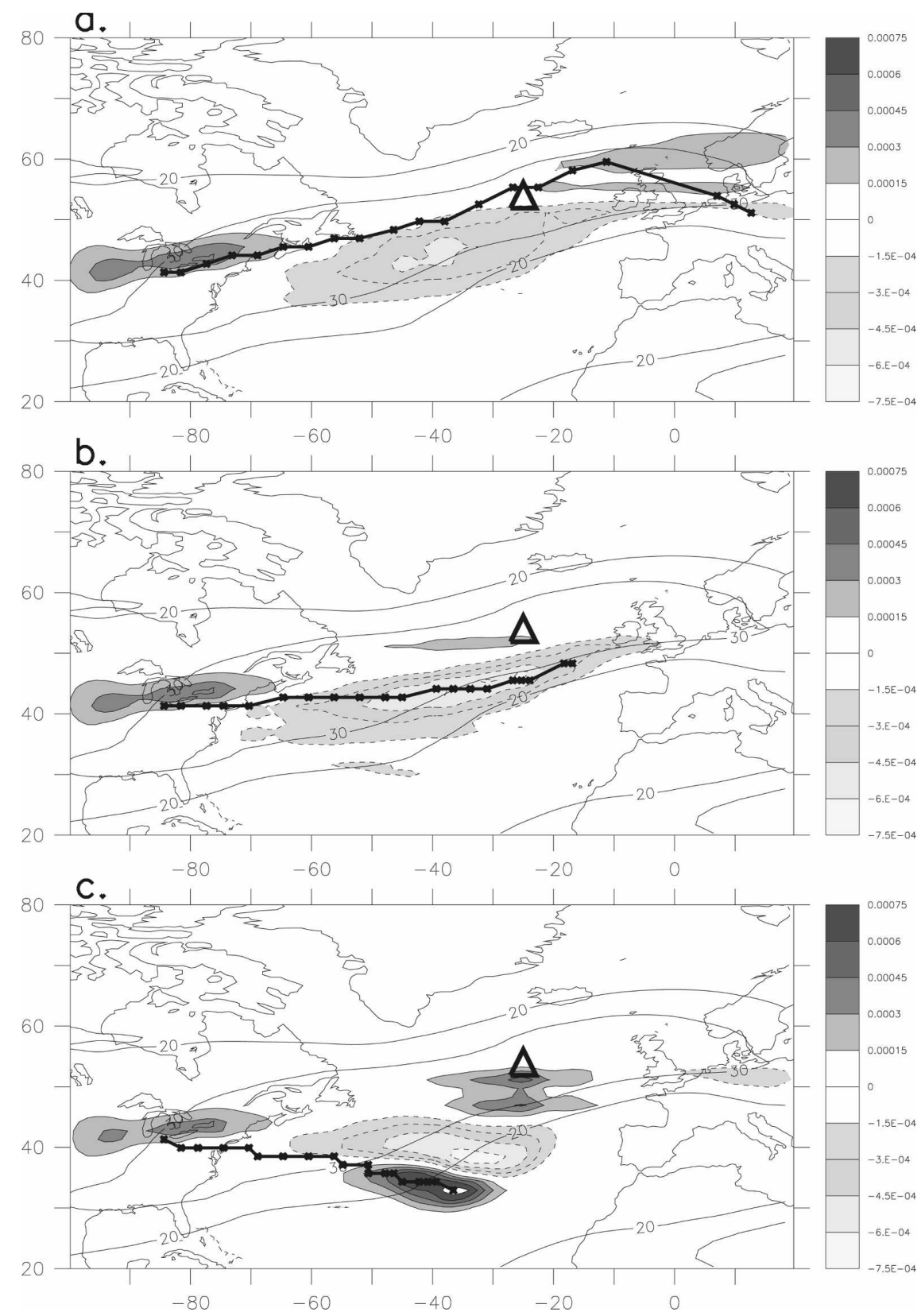

FIG. 11. Time averages of the barotropic generation rate $\mathbf{E} \cdot \overline{\mathbf{D}}$ (same shadings as in Fig. 9) for simulations starting with the cluster-4 basic state. (a) The initial perturbation streamfunction is the regression of Fig. 8 with maximum amplitude equal to $15 \times 10^{6} \mathrm{~m}^{2} \mathrm{~s}^{-1}$; (b) as in (a), but the initial perturbation is divided by 10 ; (c) as in (a), but the initial perturbation is multiplied by -1 . Heavy and thin solid lines are as in Fig. 9.

Another purpose is simply to confirm the results with a different definition for the perturbations. The perturbation streamfunction is now defined as

$$
\psi^{\prime} \equiv \psi_{0} \exp \left(-\left\{\left[\cos \left(\lambda-\lambda_{0}\right)\right]^{2}+\left(\varphi-\varphi_{0}\right)^{2}\right\}\left(\frac{a}{L}\right)^{2}\right)
$$

and is centered about the grid point $\left(\lambda_{0}, \varphi_{0}\right) ; L$ determines the length scale of the perturbation and is hereafter equal to $700 \mathrm{~km}$.

Results of simulations with different amplitudes and locations for the perturbations and different basic states are displayed in Fig. 12. In the left and right columns, the basic states are clusters 4 and 6 , respectively. Top 


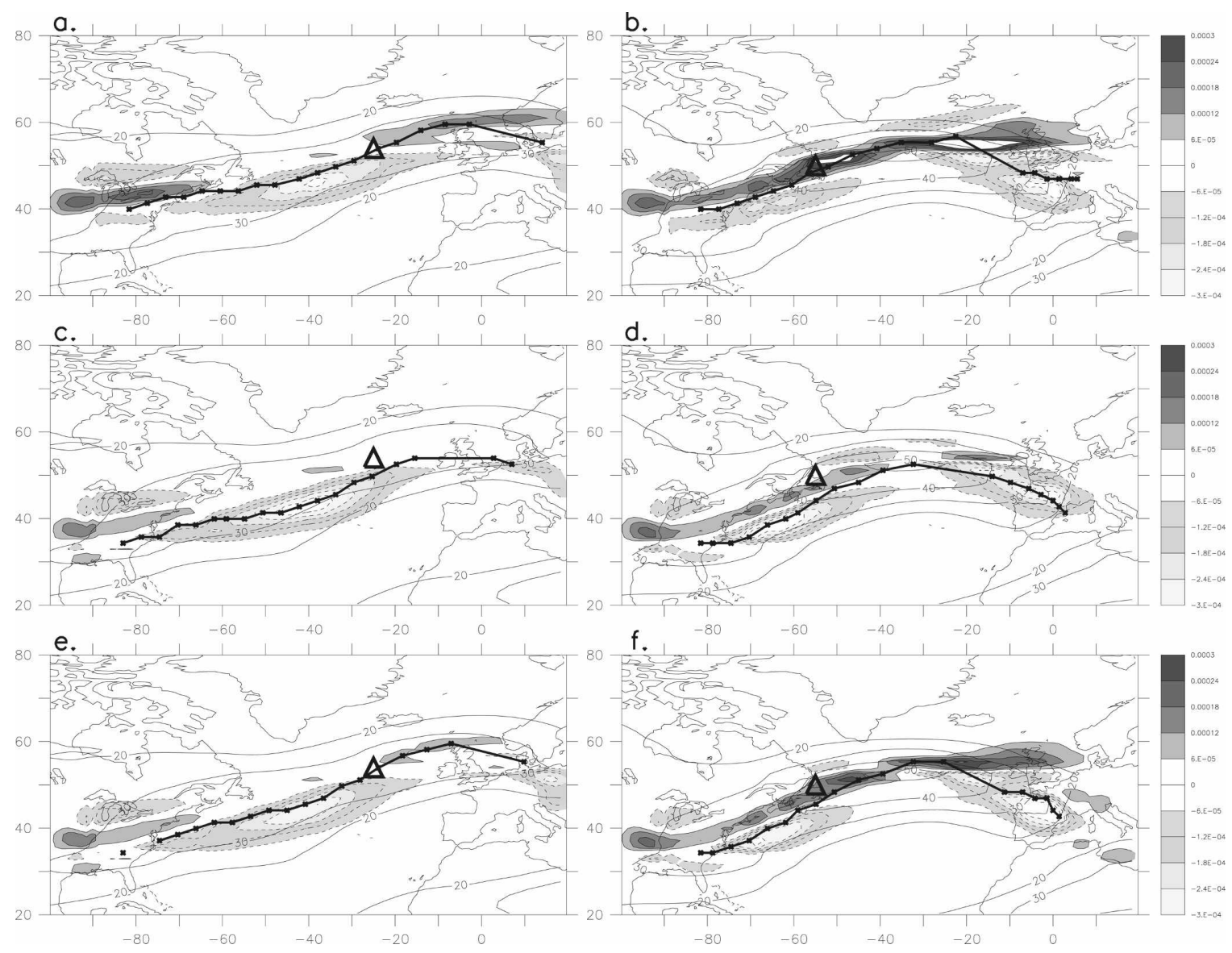

FIG. 12. Time averages of the barotropic generation rate $\mathbf{E} \cdot \overline{\mathbf{D}}$ (shaded contours; light shadings with dashed contours for negative values and dark shadings with solid contours for positive values) for simulations starting with analytically prescribed perturbations with distinct amplitudes and locations. The basic state in (a), (c), and (e) is cluster 4; in (b), (d), and (f), it is cluster 6 . The perturbation in (a) and (b) is centered about the grid point $42^{\circ} \mathrm{N}, 90^{\circ} \mathrm{W}$ and its maximum amplitude equals $15 \times 10^{6} \mathrm{~m}^{2} \mathrm{~s}^{-1}$; in (c) and (d) it is centered about $37^{\circ} \mathrm{N}, 90^{\circ} \mathrm{W}$ and its maximum amplitude equals to $15 \times 10^{6} \mathrm{~m}^{2} \mathrm{~s}^{-1}$; in (e) and (f) it is centered about $37^{\circ} \mathrm{N}, 90^{\circ} \mathrm{W}$, but its maximum amplitude is $20 \times 10^{6} \mathrm{~m}^{2} \mathrm{~s}^{-1}$. Heavy and thin solid lines are as in Fig. 9 .

panels correspond to a perturbation located at the same place $\left(\varphi_{0}=42^{\circ} \mathrm{N}, \lambda_{0}=90^{\circ} \mathrm{W}\right)$ and having the same amplitude $\left(\psi_{0}=15 \times 10^{6} \mathrm{~m}^{2} \mathrm{~s}^{-1}\right)$ as in Fig. 9, and the results are qualitatively similar. Negative and positive values of $\mathbf{E} \cdot \overline{\mathbf{D}}$ in Figs. 12a and $12 \mathrm{~b}$ are indeed located at the same place as in Figs. 9d and 9f, respectively. In middle panels, the perturbation is initially displaced southward and centered about the grid point $\left(\varphi_{0}=\right.$ $\left.37^{\circ} \mathrm{N}, \lambda_{0}=90^{\circ} \mathrm{W}\right)$ but with the same amplitude $\left(\psi_{0}=\right.$ $15 \times 10^{6} \mathrm{~m}^{2} \mathrm{~s}^{-1}$ ) as in the top panels. There are no more positive values downstream of the BtCR in Fig. $12 \mathrm{c}$ and no crossing of the jet axis of the perturbation kinetic energy maximum, in contrast with Fig. 12a. However, by keeping the same location $\left(\varphi_{0}=37^{\circ} \mathrm{N}\right.$, $\left.\lambda_{0}=90^{\circ} \mathrm{W}\right)$ and by slightly increasing the initial amplitude $\left(\psi_{0}=2010^{6} \mathrm{~m}^{2} \mathrm{~s}^{-1}\right.$; see Fig. $\left.12 \mathrm{e}\right)$, we recover positive values northeast of the BtCR similarly to Fig. 12a and a clear crossing of the BtCR by the perturba- tion. Similar results are visible when the basic state is cluster 6 (see right panels). To conclude, the more southward the initial position of the perturbation, the stronger its amplitude should be to make possible the barotropic regeneration process about the BtCR. In other words, when the perturbation is initially located far south of the jet axis, a large amplitude is essential to make possible the crossing of the jet and of the BtCR from the south to the north.

The aim is now to compare the time evolution of two distinct simulations differing only in their initial basic states. The left and right panels of Fig. 13 correspond respectively to the same simulations as in Figs. 12a,b (i.e., for which the basic states are clusters 4 and 6). They are particularly interesting to analyze together because cluster 4 presents a BtCR at the jet exit around $25^{\circ} \mathrm{W}$ whereas cluster 6 does so at the jet entrance around $55^{\circ} \mathrm{W}$. At day 1.0 , the two simulations exhibit 

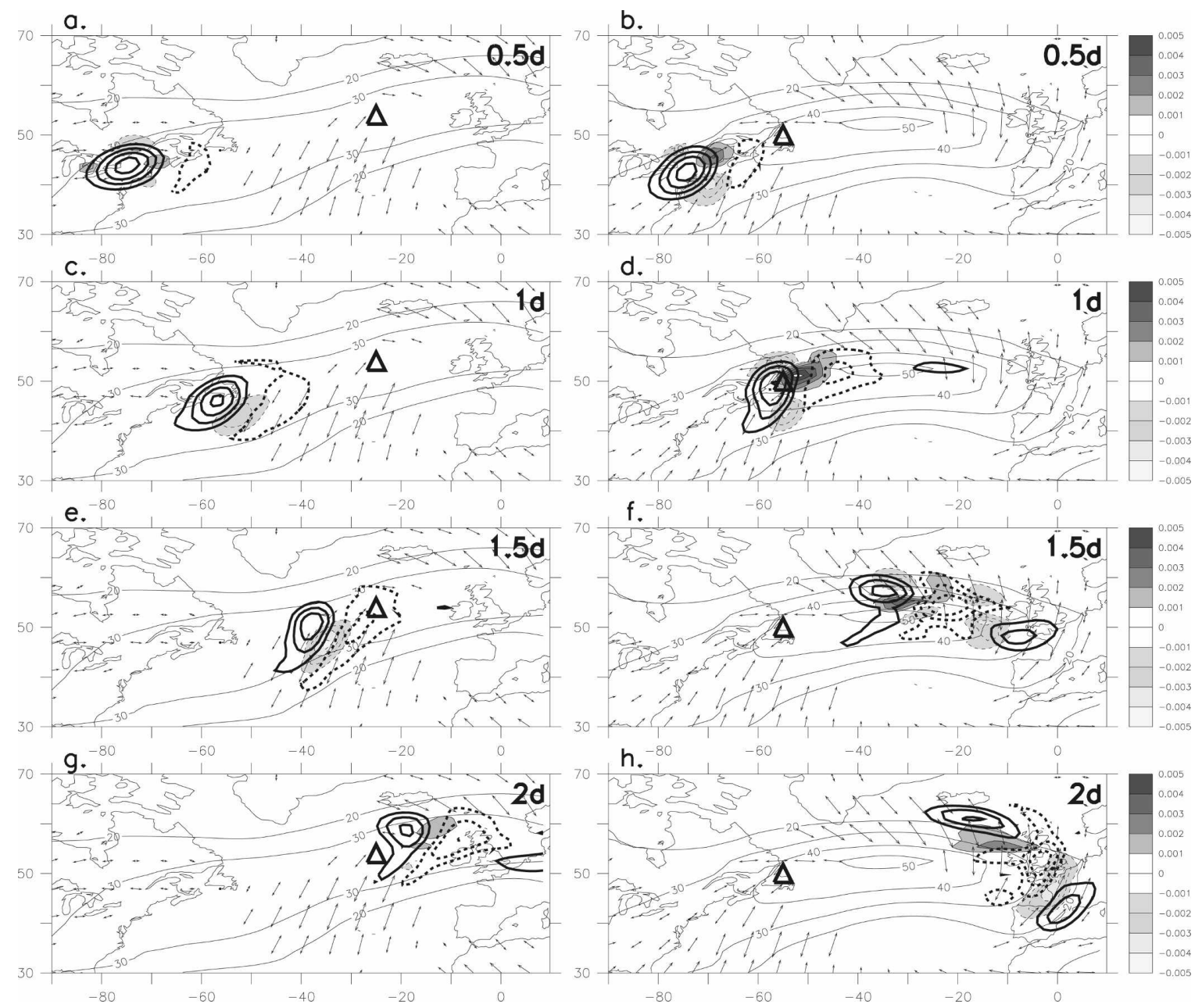

FIG. 13. Time evolution of the barotropic generation rate $\mathbf{E} \cdot \overline{\mathbf{D}}$ (shaded contours as in Fig. 10) and of the perturbation vorticity (intervals of $2.5 \times 10^{-5} \mathrm{~s}^{-1}$; heavy dashed contours for negative values and heavy solid contours for positive values) for a basic state corresponding to cluster (left) 4 and (right) 6 . The analytically prescribed perturbation for both simulations is located at $42^{\circ} \mathrm{N}, 90^{\circ} \mathrm{W}$ with maximum amplitude equal to $15 \times 10^{6} \mathrm{~m}^{2} \mathrm{~s}^{-1}$. Arrows and thin solid lines are as in Fig. 10. (a)-(b), (c)-(d), (e)-(f), and (g)-(h) correspond respectively to the flows $12,24,36$, and $48 \mathrm{~h}$ after the initial time.

distinct behavior regarding the values of the barotropic generation rate; for cluster 4 (Fig. 13c), $\mathbf{E} \cdot \overline{\mathbf{D}}$ is essentially negative whereas for cluster 6 (Fig. 13d), it is strongly positive north of the cyclone and negative south of it. At that time, in the latter case, there is already barotropic regeneration and crossing of the BtCR by the perturbation located in the western Atlantic around Newfoundland. At day 1.5, in the cluster- 4 case (Fig. 13e), the perturbation formed by a cyclone upstream and an anticyclone downstream is still entirely located upstream of the BtCR; it is strongly stretched on the anticyclonic side of the jet and $\mathbf{E} \cdot \overline{\mathbf{D}}$ is largely negative. At the same time, in the cluster- 6 case (Fig. 13f), the perturbation is essentially composed of an anticyclone and two cyclones. The more upstream cyclone corresponds to the main cyclone initialized in the beginning of the simulation, whereas the more downstream one is formed during the simulation, probably via downstream development (see Orlanski and Sheldon 1995). The former cyclone moves to the north of the jet and by day 2.0 (Fig. 13h) is entirely outside the jet axis; the latter propagates along the jet axis and is stretched in the diffluent region of the jet. This elongation explains the strong negative values of $\mathbf{E} \cdot \overline{\mathbf{D}}$ around longitude $0^{\circ}$. The negative-positive-negative pattern of the time average of $\mathbf{E} \cdot \overline{\mathbf{D}}$ in Fig. 12b is not due to an elongation-contraction-elongation of the same cyclone. The second elongation takes place for the secondary cyclone that appears and grows during the simulation. Concerning the cluster- 4 case, a barotropic regeneration stage finally happens northeast of the BtCR at day 2.0. Indeed, positive values of $\mathbf{E} \cdot \overline{\mathbf{D}}$ 


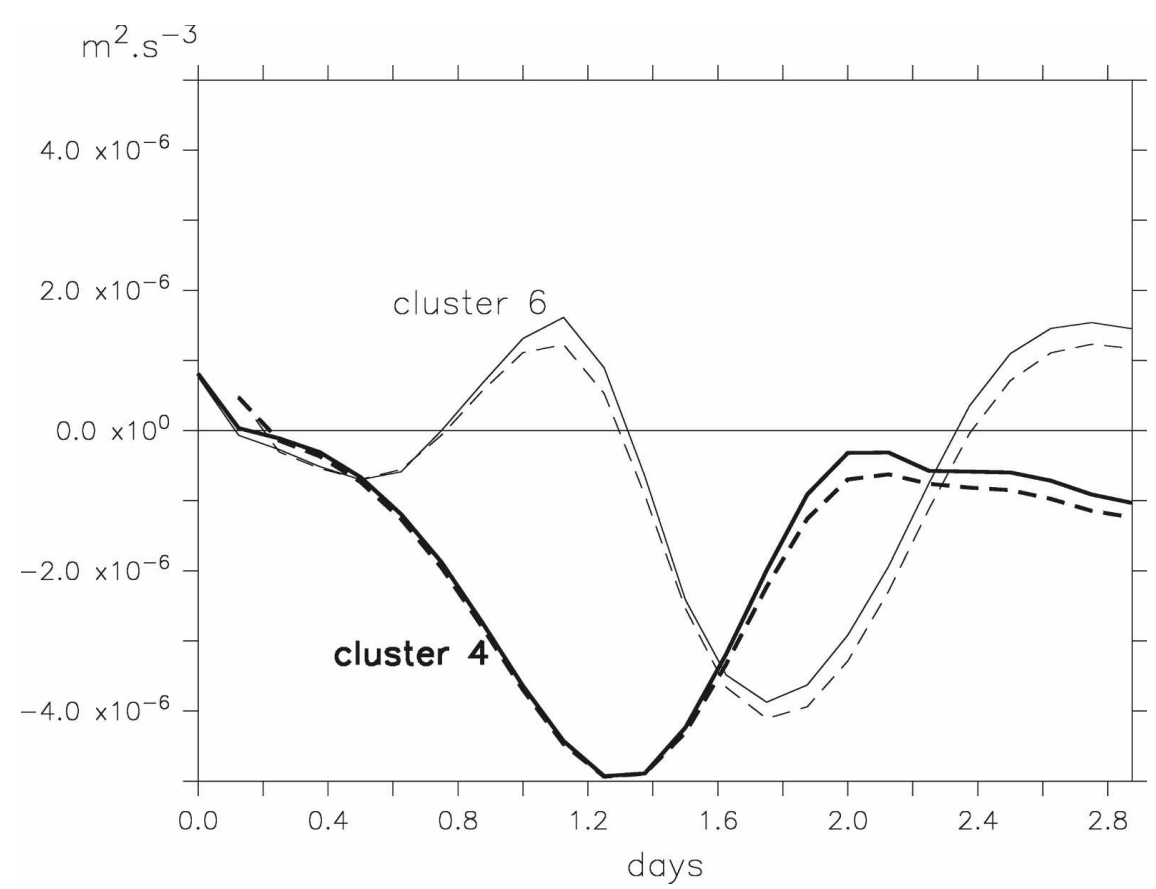

FIG. 14. Time evolution of the barotropic generation rate $\mathbf{E} \cdot \overline{\mathbf{D}}$ (solid lines) and of the eddy kinetic energy rate of change $\partial K_{e}^{\prime} / \partial t$ (dashed lines) averaged over the Northern Hemisphere for the two same simulations as in Fig. 13. Heavy and thin lines correspond to the simulations where the basic states are clusters 4 and 6 , respectively.

are clearly visible at that time (Fig. $13 \mathrm{~g}$ ), whereas there were still negative values $12 \mathrm{~h}$ before (Fig. 13e). To conclude, the same barotropic regeneration process that appears at day 1.0 in the cluster- 6 case occurs 1 day later in the cluster- 4 case and is due to the farther downstream location of the BtCR in the latter case.

The time lag between the two barotropic regeneration stages of the two different simulations is also revealed by plotting spatially averaged energy conversion rates with time in Fig. 14. First of all, we check that the curves corresponding to $\mathbf{E} \cdot \overline{\mathbf{D}}$ and to the rate of change with time of perturbation kinetic energy $\left(\partial K_{e}^{\prime} / \partial t\right)$ exhibit the same variations and reach almost the same values. This result is logical because, apart from $\mathbf{E} \cdot \overline{\mathbf{D}}$ and the diffusion term, all the other terms appearing in the perturbation kinetic energy (see RHK03) redistribute energy spatially and should not be involved in spatial averages. The peaks of $\mathbf{E} \cdot \overline{\mathbf{D}}$ and $\partial K_{e}^{\prime} / \partial t$ appear at day 1.0 and day 2.0 in the cluster- 6 and cluster- 4 cases, respectively. These peaks are related to the crossing of the BtCRs by the perturbations. Note that the peak in the cluster- 4 case is close to zero despite a clear local positive maximum of $\mathbf{E} \cdot \overline{\mathbf{D}}$ appearing at that time (Fig. $13 \mathrm{~g}$ ), but this may be due to our spatial averaging method that includes negative residues of $\mathbf{E} \cdot \overline{\mathbf{D}}$ outside the region of interest. Furthermore, although there is no global increase of perturbation kinetic energy for this particular simulation, the barotropic sink of energy is temporarily stopped during the crossing of the BtCR.

To conclude this section, the same perturbation embedded in two distinct configurations of the zonal regime can lead to very different behavior for the perturbation. The original point of our approach is that we are able for a given jet to determine a priori the area where barotropic perturbation growth may occur.

\section{3) INTERPRETATION OF NONLINEARITIES EFFECTS}

The nonlinear term involved in the perturbation vorticity equation of Eq. (4) is shown in Fig. 15a for the cluster-4 simulation after $36 \mathrm{~h}$. It has a positive maximum north of the perturbation vorticity maximum and makes the cyclone move northward. The linear term due to the advection of the basic-state absolute vorticity by the perturbation winds (Fig. 15b) is essentially negative between the cyclone and the anticyclone, which is logical because of the presence of positive absolute vorticity gradients around the jet core. The sum of the two terms shown in Fig. 15c corresponds by neglecting diffusion to the Lagrangian time derivative of the perturbation vorticity following the basic-state advection. It has a positive maximum north of the perturbation vorticity, which is only due to the nonlinear term. Hence, the nonlinear term is responsible for the northward dis- 

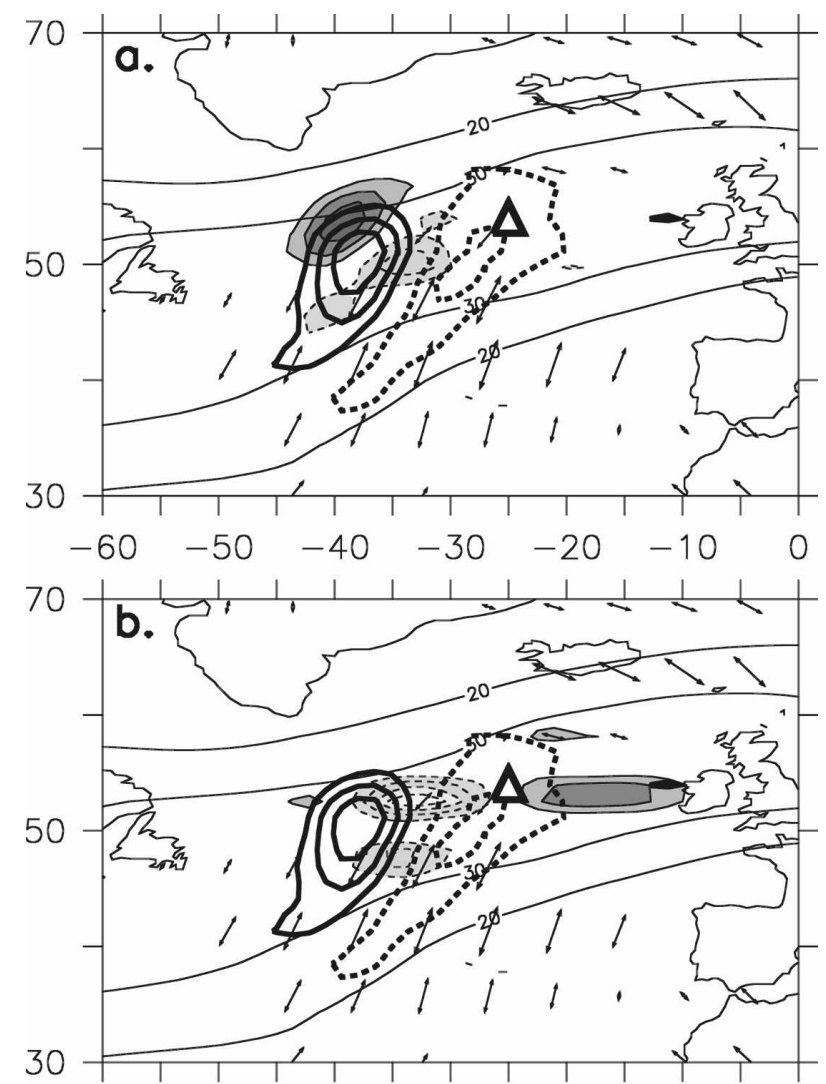
$\begin{array}{lllllll}-60 & -50 & -40 & -30 & -20 & -10 & 0\end{array}$

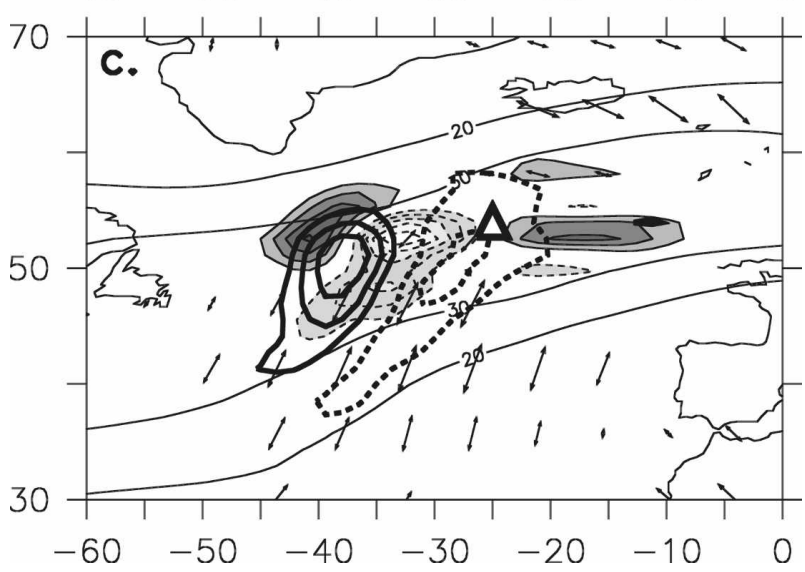

FIG. 15. Combinations of different terms involved in the perturbation vorticity equation (light shadings with dashed contours for negative values and dark shadings with solid contours for positive values; intervals of $5 \times 10^{-10} \mathrm{~s}^{-2}$ ) for the same simulation and same time as in Fig. 13e: (a) nonlinear advection $-J\left(\psi^{\prime}, \nabla^{2} \psi^{\prime}\right)$; (b) advection of the basic-state absolute vorticity by perturbation winds $-J\left(\psi^{\prime}, \nabla^{2} \bar{\psi}+f\right)$, and (c) the sum of (a) and (b). Heavy contours, thin solid lines, and double arrows denote respectively the perturbation vorticity, the basic-state wind speed, and the dilatation axes as in Fig. 13e. placement of the cyclone across the basic-state isolines and therefore for the crossing of the jet by the perturbation. This displacement can be interpreted in terms of the beta-gyre effect (McWilliams and Flierl 1979); the linear term that is due to the presence of the positive background vorticity gradient induces a meridional gyre, and the nonlinear response of this gyre is to advect the cyclones northward perpendicular to the background vorticity isolines.

In the presence of pure zonal background flows, we have checked (not shown here) that an initial cyclone located south of the jet is able to cross the jet if it has enough amplitude. Initially on the anticyclonic side of the jet, it is first stretched along the southwestnortheast direction and moves northward because of the background vorticity gradient and nonlinearities, as discussed previously. The barotropic regeneration process and the crossing of the jet from the south to the north by the cyclone can thus be reproduced in our model in the presence of pure flows (i.e., when there is no BtCR and the effective deformation is zero). However, the initial stretching of the cyclone is less strong and the regeneration process is less pronounced than in the more realistic case of nonzonal flows that are characterized by nontrivial effective deformation fields. Furthermore, in the latter case we have shown that this process is likely to occur about BtCRs. Further studies will investigate more precisely the role of the nonlinearities and their combination with the effective deformation field.

\section{Conclusions}

Barotropic dynamics of upper-tropospheric midlatitudes disturbances evolving in different kinds of zonallike jets were studied using both observational analyses and barotropic model experiments. The contraction stage of upper-level synoptic disturbances that follows their elongation stage is called barotropic regeneration process in this text. Potential areas where this process may occur are identified in the present work. These regions are called barotropic critical regions; they can be a priori located anywhere along the jet axis from the jet entrance to the jet exit depending on the horizontal inhomogeneities of the large-scale flow considered. More specifically, they are defined by horizontal maps of the effective deformation field $\Delta_{m}$. Regions where $\Delta_{m}$ is positive are important because they correspond to regions where the large-scale flow tends to strongly stretch synoptic disturbances. A BtCR is an area separating two large-scale regions of positive effective deformation, one located upstream and on the south side of the jet and the other downstream and on 
the north side of the jet. Note that the transition from the south to the north is an important ingredient of the definition of a BtCR that was not emphasized in the initial work on the subject in RJ06a but that has been revealed as playing a very important role in the present study.

In our observational analysis of the ECMWF 40-yr reanalysis dataset, all the winter days presenting a zonal weather regime are decomposed, via a partitioning algorithm, into different configurations of the effective deformation field at $300 \mathrm{hPa}$. A six-cluster partition is obtained. Composite maps of the barotropic generation rate for each cluster exhibit a succession of negative and positive values on both sides of the BtCRs and a second negative pattern far downstream of the same regions. It confirms statistically that the barotropic regeneration mechanism occurs preferentially about BtCRs, as suggested in the case studies of RJ06a. It shows also that subtle nuances exist between apparently similar zonal-like jets and make significant differences in the behavior of disturbances propagating along these jets.

Numerical experiments using a forced barotropic model on the sphere have proved that nonlinear runs are able to reproduce the barotropic regeneration process but linear ones are not. In the former case, disturbances tend to cross the jet axis from the south to the north about the BtCRs, whereas in the latter case they stay on the anticyclonic side of the jet and propagate equatorward. This is caused by the combined effects of the effective deformation and the nonlinear terms that make possible the process of barotropic regeneration about a BtCR. Note that the barotropic regeneration process does not necessarily occur with a frank crossing of the whole perturbation from the south to the north of the jet. For example, in some experiments, the perturbation vorticity maximum is around the jet axis upstream of the BtCR although most of its structure is located and stretched on the anticyclonic side of the jet.

Sensitivity experiments on the initial position, amplitude, and sign of the disturbances have been performed. In all cases shown, disturbances are essentially composed of a main vortex and other weaker vortices of opposite sign on both sides of the main vortex. The barotropic regeneration mechanism is only possible if disturbances are initially composed of a strong cyclonic vortex. Indeed, anticyclonic vortices tend to move southwestward whereas cyclonic vortices move northwestward. The more southward the initial position of the main cyclone, the stronger its amplitude should be to cross the jet about the BtCR. In some cases, because of an initial position too much in the south, the cyclone cannot be regenerated despite having a reasonable amplitude and stays in the south.

In the different experiments, the main initial vortex of the perturbation is not itself regenerated (in the sense that there is no deepening of the initial main trough). Because of downstream development, secondary vortices appear downstream of the initial vortex: it is the whole perturbation composed of the initial main vortex and other secondary vortices that is regenerated and noticeable by an increase of eddy kinetic energy. Note also that it is not the same vortex that is stretched, contracted, and finally stretched again in our simulations because the last elongation stage far downstream of the BtCR concerns the secondary cyclonic vortex and not the main one. In conclusion, it is not necessarily the same cyclone that is involved in the three-step sequence formed by the transient barotropic growth stage about a BtCR.

Climatological maps of the barotropic generation rate [e.g., those done by Lee (2000) and Black and Dole (2000)] cannot reveal the barotropic transient phase about BtCRs because their locations move from one jet to another. This is the reason why essentially negative values of $\mathbf{E} \cdot \mathbf{D}_{\mathbf{m}}$ appear over the oceans in the climatological maps. Regeneration processes detailed in Lee (2000), east of the mountains and north of the subtropical jets, are related to the climatological flow and can be reproduced by linear barotropic models. By contrast, the barotropic regeneration mechanism of the present study is intrinsically a nonlinear phenomenon. It is interesting to note, however, the common point of all the barotropic regeneration processes; they all need a discontinuity of the dilatation axes' orientation. This is true in the case of the diffluence to a confluence (e.g., east of the Rockies) and of the anticyclonic side of the extratropical jet to the cyclonic side of the subtropical jet (e.g., over North Africa), as described in Lee (2000); it is also true of the anticyclonic to the cyclonic sides of the extratropical jet, as described in the present study.

In further studies, the role of BtCRs will be investigated in the case of stratified baroclinic models. We expect that the qualitative picture will not be modified at least in the upper levels because nonlinearities will increase in presence of baroclinicity.

Acknowledgments. The author would like to thank Alain Joly for suggesting the application of the dynamical cluster algorithm to the effective deformation field and Guillaume Lapeyre for helping him in the use of the model. The paper has also benefited from discussions with Jean-Baptiste Gilet and Matthieu Plu as well 


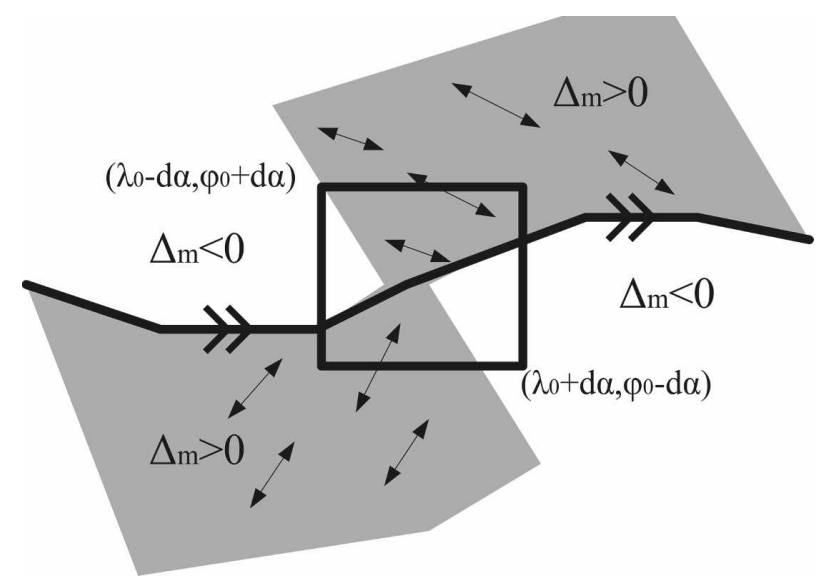

FIG. A1. Schematic of the large-scale flow configuration about a barotropic critical region and illustration of the algorithm detecting such a region. The heavy solid line with arrows indicates the jet axis. Regions of positive effective deformation and the dilatation axes inside them are represented by gray shaded areas and double arrows, respectively. The effective deformation field changes sign four times along the square of center $\left(\lambda_{0}, \varphi_{0}\right)$ and length $2 d \alpha$ represented in the figure. Furthermore, the dilatation axes' orientations averaged along the two distinct segments on the square with positive effective deformation are almost perpendicular. The algorithm considers the grid point $\left(\lambda_{0}, \varphi_{0}\right)$ as a potential candidate for a BtCR point. This process is repeated for different values of $d \alpha$ to estimate the robustness of the result (see text for more details).

as from comments and suggestions of two anonymous reviewers.

\section{APPENDIX}

\section{Automatic Detection of Barotropic Critical Regions}

The principle of the algorithm is to consider all the points along the jet core close to a separation area between two large-scale regions of positive effective deformation fields having perpendicular dilatation axes. The main steps of the algorithm are as follows: (i) At each longitude $\lambda_{0}$, we determine the latitude $\varphi_{0}$ where the background low-frequency wind speed reaches its maximum, and then (ii) a square of center $\left(\lambda_{0}, \varphi_{0}\right)$ and length $2 d \alpha$ is considered and the number of times the effective deformation field changes its sign along the square is counted. (iii) If this number is four, we determine the averaged orientations of the dilatation axes in the two segments of the square where the effective deformation is positive, and if the angle formed by these two orientations is between $60^{\circ}$ and $120^{\circ}$ (i.e., close to the orthogonality), the point $\left(\lambda_{0}, \varphi_{0}\right)$ is retained (see Fig. A1 for an illustration of such a point). (iv) If (iii) is satisfied for all the squares with $d \alpha$ varying from $6^{\circ}$ to $12^{\circ}$, the point $\left(\lambda_{0}, \varphi_{0}\right)$ is considered as being about a BtCR. The last step (iv) consists in averaging the longitudes and latitudes of all these detected points $\left(\lambda_{0}, \varphi_{0}\right)$ that are close to each other to determine the averaged position of the BtCR point. If there is no detected point between two detected ones that are $12^{\circ}$ longitude apart, they are considered as belonging to two distinct BtCR points. The choice of $12^{\circ}$ is deduced heuristically from the effective deformation fields and corresponds approximately to half the distance in degrees between the two closest BtCR points. The BtCR points detected by the algorithm (which has been applied in the Atlantic domain $\left(35^{\circ}-90^{\circ} \mathrm{N}, 90^{\circ} \mathrm{W}-0^{\circ}\right)$ for six distinct effective deformation fields) are numbered in Figs. 3a-f. We do not consider latitudes less than $35^{\circ} \mathrm{N}$ to avoid detection along the subtropical African jet; that is not the focus of the present study.

\section{REFERENCES}

Black, R., and R. Dole, 2000: Storm tracks and barotropic deformation in climate models. J. Climate, 13, 2712-2728.

Chang, E., S. Lee, and K. Swanson, 2002: Storm track dynamics. J. Climate, 15, 2163-2183.

Farrell, B., 1989: Transient development in confluent and diffluent flow. J. Atmos. Sci., 46, 3279-3288.

Hoskins, B. J., I. N. James, and G. H. White, 1983: The shape, propagation, and mean-flow interaction of large-scale weather systems. J. Atmos. Sci., 40, 1595-1612.

Lackmann, G., D. Keyser, and L. F. Bosart, 1997: A characteristic life cycle of upper-tropospheric cyclogenetic precursors during the Experiment on Rapidly Intensifying Cyclones over the Atlantic (ERICA). Mon. Wea. Rev., 125, 2729-2758.

$\_, \ldots$, and — 1999: Energetics of an intensifying jet streak during the Experiment on Rapidly Intensifying Cyclones over the Atlantic (ERICA). Mon. Wea. Rev., 127, 2777-2795.

Lee, S., 1995: Localized storm tracks in the absence of local instability. J. Atmos. Sci., 52, 977-989.

- 2000: Barotropic effects on atmospheric storm tracks. $J$. Atmos. Sci., 57, 1420-1435.

Mak, M., and M. Cai, 1989: Local barotropic instability. J. Atmos. Sci., 46, 3289-3311.

McWilliams, J., and G. Flierl, 1979: On the evolution of isolated, nonlinear vortices. J. Phys. Oceanogr., 9, 1155-1182.

Michelangeli, P.-A., R. Vautard, and B. Legras, 1995: Weather regimes: Recurrence and quasi stationary. J. Atmos. Sci., 52, 1237-1256.

Nielsen-Gammon, J., and R. Lefevre, 1996: Piecewise tendency diagnosis of dynamical processes governing the development of an upper-tropospheric mobile trough. J. Atmos. Sci., 53, 3120-3142.

Orlanski, I., and J. Sheldon, 1995: Stages in the energetics of baroclinic systems. Tellus, 47A, 605-628.

Petterssen, S., and S. Smebye, 1971: On the development of extratropical cyclones. Quart. J. Roy. Meteor. Soc., 97, 457-482.

Plaut, G., and E. Simonnet, 2001: Large-scale circulation classifi- 
cation, weather regimes, and local climate over France, the Alps, and western Europe. Climate Res., 17, 303-324.

Rivière, G., and A. Joly, 2006a: Role of the low-frequency deformation field on the explosive growth of extratropical cyclones at the jet exit. Part I: Barotropic critical region. J. Atmos. Sci., 63, 1965-1981.

and,$- 2006 \mathrm{~b}$ : Role of the low-frequency deformation field on the explosive growth of extratropical cyclones at the jet exit. Part II: Baroclinic critical region. J. Atmos. Sci., 63, 1982-1995.

, B. L. Hua, and P. Klein, 2003: Perturbation growth in terms of barotropic alignment properties. Quart. J. Roy. Meteor. Soc., 129, 2613-2635.

Sanders, F., 1988: Life history of mobile troughs in the upper westerlies. Mon. Wea. Rev., 116, 2629-2648.

Schultz, D., and F. Sanders, 2002: Upper-level frontogenesis associated with the birth of mobile troughs in northwesterly flow. Mon. Wea. Rev., 130, 2593-2610.

Vautard, R., 1990: Multiple weather regimes over the North Atlantic: Analysis of precursors and successors. Mon. Wea. Rev., 118, 2056-2081. 


\section{CORRIGENDUM}

Two press errors appeared in "Barotropic Regeneration of Upper-Level Synoptic Disturbances in Different Configurations of the Zonal Weather Regime," by Gwendal Rivière, which was published in the Journal of the Atmospheric Sciences, Vol. 65, No. 10, 3159-3178. Equation (5) should read

$$
\psi^{\prime} \equiv \psi_{0} \exp \left(-\left\{\left[\cos \varphi\left(\lambda-\lambda_{0}\right)\right]^{2}+\left(\varphi-\varphi_{0}\right)^{2}\right\}\left(\frac{a}{L}\right)^{2}\right) .
$$

Furthermore, the third sentence in the last paragraph of section $4 \mathrm{~b}(3)$ (p. 3175) should state, "The barotropic regeneration process and the crossing of the jet from the south to the north by the cyclone can thus be reproduced in our model in the presence of pure zonal flows (i.e., when there is no BtCR and the effective deformation is zero)."

The Journal of the Atmospheric Sciences regrets any inconvenience these errors may have caused. 